\title{
Late-orogenic Variscan magmatism: the case of quartz monzodiorite dykes from the Blanice Graben, southern Bohemia
}

\author{
Pozdně orogenní variský magmatismus: žíly křemenného monzodioritu \\ v blanické brázdě jižních Čech
}

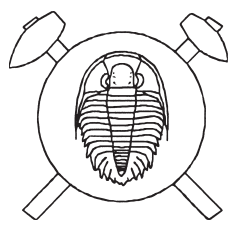

\author{
(10 figs, 5 tabs) \\ STANISLAV VRÁNA ${ }^{1 *}$ - VOJTĚCH JANOUŠEK ${ }^{1,2}$ \\ ${ }^{1}$ clo Czech Geological Survey, Klárov 3, CZ-118 21 Prague 1, Czech Republic \\ 2 Institute of Petrology and Structural Geology, Charles University, Albertov 6, CZ-128 43 Prague 2, Czech Republic \\ * Corresponding author
}

\begin{abstract}
Pyroxene-biotite quartz monzodiorite of the Štěpánovice dyke (3 km long and up to $50 \mathrm{~m}$ wide), together with additional small occurrences further north, is associated with the Late Variscan Blanice Graben in the Moldanubian Zone of southern Bohemia. The texture is subophitic, fine- to medium-grained, with skeletal crystals of ilmenite and zircon. This, together with rare amygdaloidal texture, indicates a rapid cooling of the melt emplaced at shallow (subvolcanic) crustal levels. Quartz monzodiorites are metaluminous $(\mathrm{A} / \mathrm{CNK}=0.79-0.86)$, high$\mathrm{K}$ calc-alkaline rocks straddling the boundary to the shoshonitic domain. A characteristic feature in the Primitive-Mantle normalized spider plots are LREE/HREE enrichments as well as $\mathrm{Nb}-\mathrm{Ti}-\mathrm{P}$ troughs, resembling magmas derived in continental arc settings. The Nd isotopic compositions of two samples $\left(\varepsilon_{290}^{\mathrm{Nd}} \sim-2.6\right.$ and -3.1$)$ correspond to single-stage $\mathrm{Nd}$ depleted-mantle model ages of 0.97 and $1.02 \mathrm{Ga}$; the strontium is unradiogenic $\left({ }^{87} \mathrm{Sr}^{86} \mathrm{Sr}_{290}=0.7064\right.$ and 0.7066$)$. The studied quartz monzonite dykes show a geological position, petrologic and whole-rock geochemical affinity to pyroxene microgranodiorite-quartz monzodiorite of the adjacent, $\sim 270$ Ma old Ševětín dyke swarm and the quartz micromonzodioritic Sušice dyke swarm further $75 \mathrm{~km}$ to the WNW. All these late Variscan (?Permian) basic-intermediate magmas seem to have intruded in extensional regime, following the orogenic collapse. The attendant crustal thinning could have led to upwelling and adiabatic decompression melting of the local, by Late Palaeozoic subduction-modified, lithospheric mantle. The Sr-Nd isotopic data point to assimilation of crustal material by rather primitive, slightly enriched mantle-derived magmas.
\end{abstract}

Key words: quartz monzodiorite; petrology; geochemistry; petrogenesis; Bohemian Massif; Variscan orogeny

\section{Introduction}

The waning stages of the Variscan orogeny in Central Europe were accompanied by development of intermontane basins (see Mattern 2001 for review) and the Late Carboniferous-Permian syn-sedimentary magmatic activity, often with a distinct calc-alkaline chemistry and rather primitive $\mathrm{Sr}-\mathrm{Nd}$ isotopic signature (Schmidberger Hegner 1999; Romer et al. 2001; Ulrych et al. 2006). However, analogous igneous rocks are also widespread in the Variscan foreland (e.g., Benek et al. 1996).

While volcanics occurring in the Sudetic, Central and Western Bohemian basins in the Czech Republic have been a subject of recent investigations (Ulrych et al. 2006 and references therein), no attention so far has been paid to the igneous activity within the Moldanubian Unit. Here, the magmatism is connected mainly with the Blanice Graben, a major NNE-SSW trending Late Variscan structure, running from the area east of Prague towards České Budějovice (Fig. 1a). The graben is filled by a sequence of downfaulted Stephanian and Permian sediments, up to c. $500 \mathrm{~m}$ thick. The regional extent of the exposed part of the Blanice Graben (c. $200 \mathrm{~km})$, together with sinistral displacement near $17 \mathrm{~km}$ indicated in the Kaplice area (Vrána - Bártek 2005), suggest that the fault zone probably tapped deep structures near the crust-upper mantle boundary. Still, no lava flows or tuff horizons were recorded from its sedimentary fill.
Previously, seven dykes (termed Ševětín dyke swarm here) of pyroxene microgranodiorite to amphibole-pyroxene quartz micromonzonite were characterized as shallow-level (subvolcanic) intrusions of Autunian age (Vrána et al. 1993, Košler et al. 2001). They crop out in the area between Veselí nad Lužnicí and České Budějovice. The microgranodiorite dykes were emplaced mainly along local, NE-SW trending faults, which probably are second-order faults, oblique in relation to the $\mathrm{N} 10^{\circ} \mathrm{E}$ fault zone of the Blanice Graben. Analogous suite of quartz monzodioritic Sušice dykes intruded mainly along a N-S trending fault zone south of the Central Bohemian Plutonic Complex, c. 50 NW of České Budějovice.

The present study deals with Late Variscan (?Permian) subvolcanic quartz monzodiorite dykes, intimately associated with the tectonic zone of the Blanice Graben. These comprise the main Štěpánovice dyke (E of České Budějovice) and smaller occurrences (Hamr and Čítov) further to the NNE (Fig. 1b-c). The quartz monzodiorites attracted attention owing to their rather primitive, high-K calc-alkaline/shoshonitic chemistry, with low $\mathrm{Sr}$ isotopic ratios and unradiogenic $\mathrm{Nd}$, translating to low $\mathrm{Nd}$ model ages. The current paper represents a contribution to the Late Variscan development of the Moldanubian Zone of the Bohemian Massif, as well as causes and sources of late orogenic high-K calc-alkaline magmatism in general. 


\section{Geological setting}

Mafic dykes associated with the Blanice Graben

The Štěpánovice dyke of pyroxene-biotite quartz monzodiorite, trending $\mathrm{N}-\mathrm{S}$, crops out in the eastern part of the Lišov granulite Massif in southern Bohemia (Fig. 1b). The dyke, $3 \mathrm{~km}$ long and 20-50 m wide, cuts the local two-pyroxene mafic granulites and migmatites. Quartz monzodiorite of this dyke was exploited for decades in the Skalice quarry as a dimension stone. There is another small, disused quarry east of the Vlkovice village (Fig. 1b).

Additional occurrences of quartz monzodiorite with comparable geochemistry and petrology are known from the vicinity of Veselí nad Lužnicí ( $25 \mathrm{~km}$ to the NNE), namely from the village of Hamr and the Čítov forest (Fig. 1c). At these two localities the quartz monzodiorite was sampled in loose blocks up to $0.5 \mathrm{~m}$ in size. The dominant country rock type in this area is sillimanitecordierite-biotite migmatite.

\section{Geochronology}

The available geochronological data point to episodic yet long-lived tectonic activity of the Blanice Graben fault zone (including its southern part, i.e. the Kaplice-Rödl Fault):

- low-T muscovite (sericite) crystallized in the KapliceRödl Fault Zone mylonites was dated by the Ar-Ar method at 288-281 Ma (Brandmayr et al. 1995),

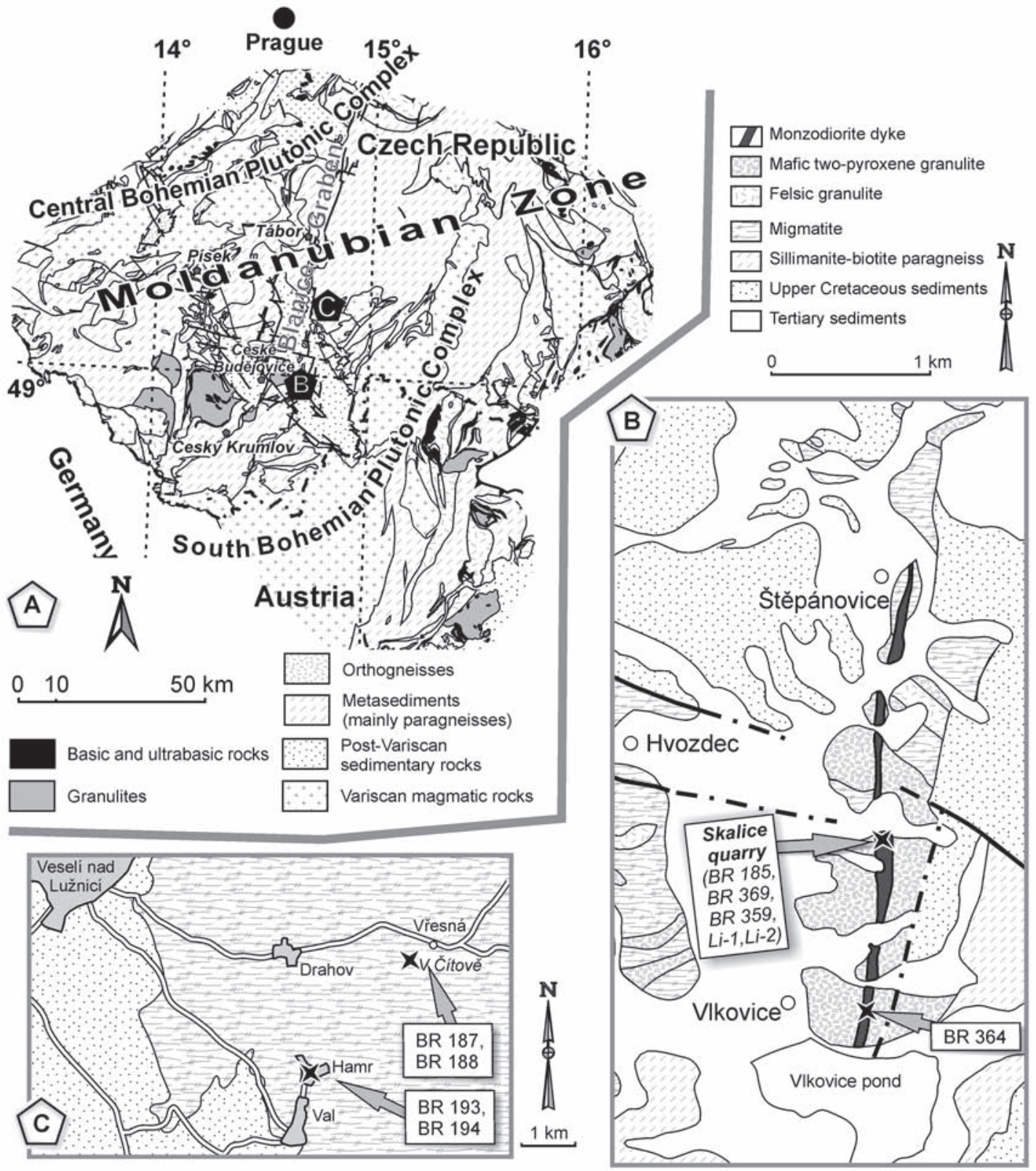

Fig. $1 \mathrm{a}$ - Location of the studied quartz monzodiorite occurrences (labelled ' $\mathrm{B}$ ' and ' $\mathrm{C}$ ') within the Moldanubian Zone of the Bohemian Massif. Simplified from the Czech Geological Survey map 1:500 000; b - Geological setting of the Štépánovice dyke (based on the Czech Geological Survey map 1:25 000, sheet Lišov); c - Schematic map of the mafic dyke outcrops SE of Veselí nad Lužnicí. 
- the primary magmatic amphibole (titanian pargasite) from microgranodiorite/quartz monzonite dyke of the Ševětín swarm (Dehetník, NNE of České Budějovice; Vrána et al. 1993) yielded an $\mathrm{Ar}-\mathrm{Ar}$ age of $270 \pm 2 \mathrm{Ma}$ (Košler et al. 2001)

- hornblende from quartz diorite dyke of the Kaplice swarm has been dated at $303 \pm 5 \mathrm{Ma}$ by the $\mathrm{Ar}-\mathrm{Ar}$ method (Vrána et al. 2005)

In addition, the latest plutonic suite of the South Bohemian Plutonic Complex (Mauthausen Group: Gerdes, 1997) shows signs of emplacement localized to some extent along the late Variscan brittle fault structures, as indicated especially by the Kaplice dyke swarm of biotite granodiorite porphyries (Vrána et al. 2005) and the so-called Pfahl granite in Austria (Gerdes 1997). Within the Mauthausen Group, the Freistadt granodiorite has been dated by $\mathrm{U}-\mathrm{Pb}$ method on monazite at $302 \pm 2 \mathrm{Ma}$ (Friedl et al. 1992) and similar within the error age yielded the Rb-Sr WR dating of the Pfahl granite (312 \pm 9 Ma: Gerdes 1997). Young age of the monazite from the Mauthausen granite was confirmed recently by conventional $\mathrm{U}-\mathrm{Pb}$ dating $(316 \pm 1 \mathrm{Ma}$ : Gerdes et al. 2003).

\section{Analytical techniques}

\section{Electron microprobe}

Analyses were carried out with the CamScan 3400 electron microscope using energy dispersion analyzer Link ISIS at the Czech Geological Survey, Prague-Barrandov. Analytical conditions were $2.5 \mathrm{nA}, 15 \mathrm{kV}, 60 \mathrm{~s}$ counting time on sample, $120 \mathrm{~s}$ on standard (J. Malec, analyst).

\section{Whole-rock geochemistry}

Most of the major-element whole-rock analyses were performed by wet chemical methods in the laboratories of the Czech Geological Survey, Prague-Barrandov. The trace elements were analysed in the ACME Analytical Laboratories, Vancouver, Canada. The majority of the trace elements were determined by $\mathrm{LiBO}_{2} / \mathrm{Li}_{2} \mathrm{~B}_{4} \mathrm{O}_{7}$ fusion and ICP-MS/ES, except for precious and base metals analysed by aqua regia digestion followed by ICP-MS. Data management, recalculation, plotting and statistical evaluation of the data were facilitated using the $\mathrm{R}$ language package GCDkit (Janoušek et al. 2006a).

\section{Radiogenic isotopes}

For the isotopic study, samples were dissolved using a combined $\mathrm{HF}-\mathrm{HCl}-\mathrm{HNO}_{3}$ attack. Strontium and bulk REE were isolated by standard cation-exchange chromatography techniques on quartz columns with BioRad AG50Wx8 resin, Nd was further separated on quartz columns with Biobeads S-X8 coated with HDEHP (Richard et al. 1976). Isotopic analyses were performed on Finnigan MAT 262 thermal ionization mass spectrometer in static mode using a double Re filament assembly (CGS). The ${ }^{143} \mathrm{Nd} /{ }^{144} \mathrm{Nd}$ ratios were corrected for mass fractionation to ${ }^{146} \mathrm{Nd} /{ }^{144} \mathrm{Nd}=0.7219,{ }^{87} \mathrm{Sr} /{ }^{86} \mathrm{Sr}$ ratios assuming ${ }^{86} \mathrm{Sr} /{ }^{88} \mathrm{Sr}=0.1194$. External reproducibility is given by results of repeat analyses of the La Jolla $\left({ }^{143} \mathrm{Nd} /{ }^{144} \mathrm{Nd}=\right.$ $0.511858 \pm 26(2 \sigma), \mathrm{n}=18)$ and NBS $987\left({ }^{87} \mathrm{Sr} /{ }^{86} \mathrm{Sr}=\right.$ $0.710250 \pm 24(2 \sigma), \mathrm{n}=10)$ isotopic standards. The $\mathrm{Rb}$, $\mathrm{Sr}, \mathrm{Sm}$ and Nd concentrations were obtained by ICP-MS in ACME Analytical Laboratories, Canada.

The decay constants applied to age-correct the isotopic ratios are from Steiger - Jäger (1977) (Sr) and Lugmair et al. (1978) $(\mathrm{Nd})$. The $\varepsilon_{\mathrm{Nd}}^{\mathrm{i}}$ values and single-stage CHUR Nd model ages were obtained using Bulk Earth parameters of Jacobsen - Wasserburg (1980), the twostage Depleted Mantle Nd model ages $\left(\mathrm{T}_{\mathrm{DM}}^{\mathrm{Nd}}\right)$ were calculated after Liew - Hofmann (1988).

\section{Field relations}

The quartz monzodiorite is a massive, fine-grained or locally medium-grained rock of grey colour (Fig. 2). Biotite flakes up to 1-3 $\mathrm{mm}$ and the light-coloured plagioclase aggregates dominate the megascopic aspect. The samples from the Hamr and Č́tov localities are finer grained than those from the Štěpánovice dyke. Quartz monzodiorite shows numerous heterogeneities and enclaves, studied mainly in the well-exposed Štépánovice dyke:

(i) somewhat irregular chlorite-actinolite spheroids, mainly 3 to $5 \mathrm{~mm}$ in diameter, accounting for estimated 3 to $5 \%$ of the rock volume (Fig. 2). The chlorite with $\mathrm{Mg} 3.03$ and $\mathrm{Fe} 1.77$ atoms per formula unit (apfu) is intergrown with acicular actinolite crystals

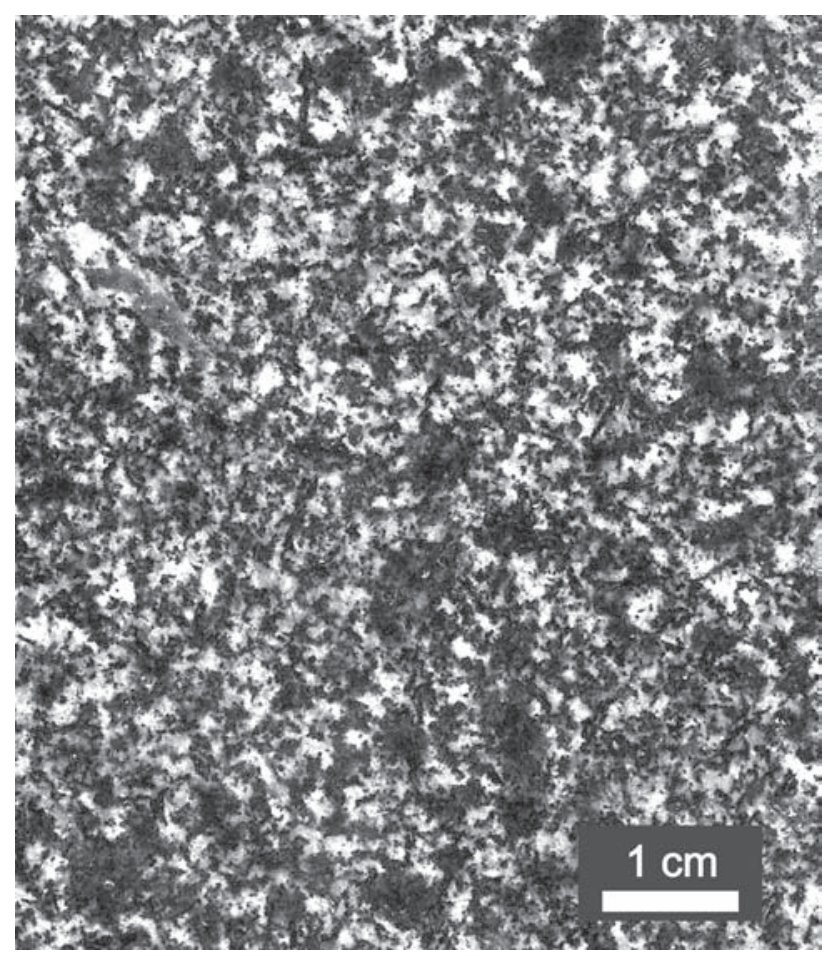

Fig. 2 Macroscopic appearance of the pyroxene-biotite quartz monzodiorite in a polished slab (Štěpánovice dyke, Skalice quarry). 
(less than 5 vol. \% of the spheroid), making a regular c. $60^{\circ}$ network;

(ii) rather rare, up to several $\mathrm{mm}$ long Al-rich inclusions with hercynite, corundum \pm diaspore and relict sillimanite set in a matrix rich in plagioclase and secondary muscovite;

(iii) amygdales up to $6 \mathrm{~mm}$ in diameter, filled by calcite, quartz and K-feldspar, were encountered only in exploration borehole drilled in 1960s next to the Skalice quarry;

(iv) abundant xenoliths of white hydrothermal quartz, $1-20 \mathrm{~cm}$ in size, amounting up to 20 enclaves per $\mathrm{m}^{2}$. The quartz xenoliths are rimmed by a reaction zone of diopsidic clinopyroxene and K-feldspar in micrographic intergrowths with quartz;

(v) scarce igneous enclaves; a) leucogranitic, represented by analysis BR 359 (Tables 3-4), and b) still rarer enclaves of leucocratic quartz monzodiorite, such as a $6 \mathrm{~cm}$ long fragment dominated by euhedral plagioclase, accompanied by K-feldspar and quartz, as well as minor biotite. The latter probably corresponds to an early-crystallized material, brought up by the melt.

In addition, some of the quartz monzodiorites were contaminated by incompletely digested xenolith material. For instance the sample BR 188 from Čítov contains relics of a strongly resorbed feldspar-rich rock.

\section{Petrology and mineral chemistry}

Characteristic of the quartz monzodiorites is the subophitic texture, whereby early-crystallized euhedral plagioclase laths are enclosed in augite oikocrysts (Fig. 3b). Moreover, locally present are micrographic intergrowths of K-feldspar with quartz that formed at the terminal stage of the crystallization. The overview of the mineral assemblage present in the quartz monzodiorite dykes is given in Table 1.

Table 1 Mineralogy of the pyroxene-biotite quartz monzodiorites.

\begin{tabular}{|llll|}
\hline $\begin{array}{l}\text { Primary } \\
\text { minerals }\end{array}$ & Sulphides & Al-contamination & $\begin{array}{l}\text { Secondary } \\
\text { minerals }\end{array}$ \\
\hline ilmenite & pyrrhotite & sillimanite & titanite \\
zircon & pentlandite & hercynite & epidote \\
baddeleyite & Ni-cobaltite & $\begin{array}{l}\text { corundum } \\
\text { actinolite }\end{array}$ & chlorite \\
allanite & gersdorffite & $\begin{array}{l}\text { pseudomorphs } \\
\text { after magnetite }\end{array}$ & prehnite \\
monazite & chalcopyrite & diaspore & sericite \\
apatite & sphalerite & & calcite \\
low-Ca augite & galena & & \\
high-Ca augite & pyrite & & \\
plagioclase & & & \\
biotite & & & \\
amphibole & & & \\
K(Na)-feldspar & & & \\
quartz & & & \\
\hline
\end{tabular}

Plagioclase

Plagioclase forms 1) nearly equant, subhedral to euhedral crystals 0.5 to $1 \mathrm{~mm}$ across, and 2) somewhat smaller, euhedral laths $0.5 \mathrm{~mm}$ long. For the first type is characteristic normal zoning; the cores are andesine to calcic labradorite, from which the $\mathrm{Ca}$ declines rather steeply towards the albite-oligoclase or albite rims (Table 2). For instance, a fresh crystal Pl a (Table 2) was calcic labradorite in the core $\left(\mathrm{An}_{65.8}\right)$ and albite-oligoclase at the rim $\left(A n_{11.3}\right)$. A relatively early generation of plagioclase is represented by rare fractured inclusions of a calcic plagioclase, enclosed in undeformed andesine-labradorite core domains (Fig. 3c). The smaller tabular plagioclase crystals of the second type (e.g., Pl b in Table 2) are andesine $\left(\mathrm{An}_{42.8}\right)$ in the centre and albite $\left(\mathrm{An}_{6.6}\right)$ at the rim.

Many plagioclase crystals are "filled" by alteration products. For instance, the core part of a $1 \mathrm{~mm}$ long crystal is a three-phase mosaic composed of calcic labradorite $\mathrm{An}_{66.1}$ (probably relics of the original feldspar), muscovite and barian potassic feldspar $\left(\mathrm{Ab}_{3.2} \mathrm{Cn}_{11.4}\right.$, Table 2$)$. The individual compositional domains are of the order of ten microns. It is uncertain, whether the association of barian K-feldspar with labradorite (and muscovite) in the altered core indicates a former presence of a ternary feldspar, modified by unmixing and a probable alteration indicated by muscovite. Rare prehnite may be present in the core.

\section{Pyroxene}

Clinopyroxene crystals in the quartz monzodiorite of the Štěpánovice dyke are usually $0.2-0.7 \mathrm{~mm}$ across. The mineral contains $0.9-1.8$ wt. $\% \mathrm{TiO}_{2}, 2.4-4.4$ wt. \% $\mathrm{Al}_{2} \mathrm{O}_{3}, 0.0-0.8$ wt. $\% \mathrm{Cr}_{2} \mathrm{O}_{3}$, with $\mathrm{Mg} 0.88$ and $\mathrm{Fe} 0.24$ (apfu). Some of the crystals are, at their margins, replaced by magnesiohornblende (low-Al), grass green in plane polarized light if enriched in Cr. The sample BR 194 from Hamr contains low-Ca augite with 6.0 to 6.6 wt. \% $\mathrm{CaO}$ (corresponding to former pigeonite), in addition to ordinary, high-Ca augite. Quartz monzodiorite samples contain no safely identifiable relics of orthopyroxene. However some chlorite aggregates, grading to spherules, show textures similar to "bastite" pseudomorphs after orthopyroxene. It is thus possible that the quartz monzodiorites contained up to three different pyroxenes in initial stages of their crystallization. The crystallization of the two or three types of pyroxene could have been sequential and a xenocrystic character of the early orthopyroxene cannot be excluded (compare with the situation in the Sušice dykes, Vrána 2004).

\section{Biotite}

Biotite crystals, typically $0.3-2 \mathrm{~mm}$ (up to $3-5 \mathrm{~mm}$ ) long, are often strongly affected by chloritization. Considerably less common is a local replacement by epidote or prehnite. The titanium content is rather high (up to $6.3 \mathrm{wt} . \%$ 
Table 2 Representative analyses of minerals in pyroxene-biotite quartz monzodiorite.

\begin{tabular}{|c|c|c|c|c|c|c|c|c|c|c|c|c|}
\hline $\begin{array}{l}\text { Analysis No. } \\
\text { Mineral* }\end{array}$ & $\begin{array}{c}3 \\
\text { High-Ca } \\
\text { Augite }\end{array}$ & $\begin{array}{c}9 \\
\text { Biotite }\end{array}$ & $\begin{array}{c}4 \\
\text { Ilmenite }\end{array}$ & $\begin{array}{r}23 \\
\text { Chlorite }\end{array}$ & $\begin{array}{c}24 \\
\text { Acti- } \\
\text { nolite }\end{array}$ & $\begin{array}{r}12 \\
\text { Pl a } \\
\text { core }\end{array}$ & $\begin{array}{c}15 \\
\text { Pl a } \\
\text { rim }\end{array}$ & $\begin{array}{c}16 \\
\text { Pl b } \\
\text { core }\end{array}$ & $\begin{array}{c}17 \\
\text { Pl b } \\
\text { rim }\end{array}$ & $\begin{array}{r}21 \\
\text { Pl c }\end{array}$ & $\begin{array}{c}20 \\
\text { Kfs (Ba) }\end{array}$ & $\begin{array}{c}25 \\
\text { Spinel }\end{array}$ \\
\hline $\mathrm{SiO}_{2}$ & 51.79 & 35.61 & 0.08 & 28.31 & 56.17 & 52.37 & 66.56 & 57.68 & 68.04 & 51.53 & 60.54 & n.a. \\
\hline $\mathrm{TiO}_{2}$ & 0.87 & 6.29 & 48.50 & n.a. & n.a. & n.a. & n.a. & n.a. & n.a. & n.a. & n.a. & 0.33 \\
\hline $\mathrm{Al}_{2} \mathrm{O}_{3}$ & 2.41 & 13.99 & 0.05 & 18.38 & 1.14 & 30.31 & 21.30 & 27.00 & 20.50 & 30.30 & 19.60 & 60.81 \\
\hline $\mathrm{Cr}_{2} \mathrm{O}_{3}$ & 0.38 & 0.11 & 0.28 & n.a. & n.a. & n.a. & n.a. & n.a. & n.a. & n.a. & n.a. & 0.00 \\
\hline $\mathrm{FeO}_{\mathrm{t}}$ & 8.21 & 18.91 & 48.35 & 20.63 & 12.14 & 0.51 & 0.05 & 0.52 & 0.41 & 0.48 & 0.02 & 27.34 \\
\hline MnO & 0.35 & 0.15 & 2.18 & 0.10 & 0.30 & n.a. & n.a. & n.a. & n.a. & n.a. & n.a. & 0.07 \\
\hline $\mathrm{MgO}$ & 15.91 & 10.92 & 0.19 & 19.79 & 16.56 & 0.03 & 0.00 & 0.00 & 0.00 & n.a. & n.a. & 11.77 \\
\hline $\mathrm{ZnO}$ & n.a. & n.a. & n.a. & n.a. & n.a. & n.a. & n.a. & n.a. & n.a. & n.a. & n.a. & 0.89 \\
\hline $\mathrm{CaO}$ & 19.56 & 0.00 & 0.04 & 0.05 & 12.99 & 13.41 & 2.38 & 9.03 & 1.41 & 13.77 & 0.00 & n.a. \\
\hline $\mathrm{Na}_{2} \mathrm{O}$ & 0.23 & 0.28 & n.a. & 0.24 & 0.14 & 3.66 & 9.85 & 6.42 & 11.02 & 3.56 & 0.34 & n.a. \\
\hline $\mathbf{K}_{2} \mathbf{O}$ & n.a. & 9.18 & n.a. & n.a. & n.a. & 0.29 & 0.67 & 0.38 & 0.05 & 0.44 & 13.99 & n.a. \\
\hline $\mathrm{BaO}$ & n.a. & n.a. & n.a. & n.a. & n.a. & n.a. & n.a. & n.a. & n.a. & 0.26 & 6.10 & n.a. \\
\hline Total & 99.71 & 95.44 & 99.67 & 87.50 & 99.44 & 100.58 & 100.81 & 101.03 & 101.43 & 100.34 & 100.59 & 101.21 \\
\hline $\begin{array}{l}\text { Number of } \\
\text { cations or } \\
(\mathrm{O}+\mathrm{OH}) \text { anions }\end{array}$ & 4 & $24(\mathrm{O}+\mathrm{OH})$ & 4 & $18(\mathrm{O}+\mathrm{OH})$ & $24(\mathrm{O}+\mathrm{OH})$ & 20 & 20 & 20 & 20 & 20 & 20 & 3 \\
\hline Si & 1.921 & 5.445 & 0.004 & 2.909 & 7.902 & 9.488 & 11.656 & 10.250 & 11.783 & 9.372 & 11.581 & \\
\hline $\mathbf{A l}^{\mathrm{IV}}$ & 0.079 & 2.521 & 0.003 & 1.091 & 0.098 & 6.472 & 4.396 & 5.655 & 4.184 & 6.495 & 4.419 & 1.902 \\
\hline $\mathbf{A l}^{\mathrm{VI}}$ & 0.025 & & & 1.135 & 0.090 & & & & & & & \\
\hline $\mathbf{T i}$ & 0.024 & 0.723 & 1.837 & & & & & & & & & 0.007 \\
\hline $\mathrm{Cr}$ & 0.011 & 0.013 & 0.011 & & & & & & & & & \\
\hline $\mathrm{Fe}^{3+}$ & 0.010 & 0.000 & 0.305 & & 0.000 & & & 0.143 & & 0.118 & & 0.085 \\
\hline $\mathrm{Fe}^{2+}$ & 0.244 & 2.418 & 1.731 & 1.773 & 1.428 & 0.077 & 0.007 & & 0.059 & & 0.003 & 0.522 \\
\hline Mn & 0.011 & 0.019 & 0.092 & 0.009 & 0.036 & & & & & & & 0.002 \\
\hline Mg & 0.880 & 2.489 & 0.014 & 3.032 & 3.473 & 0.008 & & & & & & 0.466 \\
\hline $\mathrm{Zn}$ & & & & & & & & & & & & 0.018 \\
\hline $\mathrm{Ca}$ & 0.777 & & 0.002 & 0.006 & 1.958 & 2.603 & 0.447 & 1.719 & 0.262 & 2.683 & 0.00 & \\
\hline $\mathbf{N a}$ & 0.017 & 0.083 & & 0.047 & 0.038 & 1.286 & 3.344 & 2.212 & 3.700 & 1.255 & 0.126 & \\
\hline $\mathbf{K}$ & & 1.791 & & & & 0.067 & 0.150 & 0.086 & 0.011 & 0.102 & 3.414 & \\
\hline $\mathbf{B a}$ & & & & & & & & & 0.018 & 0.457 & & \\
\hline An & & & & & 65.81 & 11.33 & 42.80 & 6.59 & 66.10 & 0.00 & & \\
\hline $\mathbf{A b}$ & & & & & 32.50 & 84.87 & 55.06 & 93.14 & 30.93 & 3.15 & & \\
\hline Or & & & & & 1.69 & 3.80 & 2.14 & 0.28 & 2.52 & 85.41 & & \\
\hline Cn & & & & & & & & & 0.46 & 11.44 & & \\
\hline
\end{tabular}

* Explanations: Pl plagioclase, Kfs K-feldspar. All analyses are from sample Li-2

$\left.\mathrm{TiO}_{2}\right) ; \mathrm{Mg}=2.49$ and $\mathrm{Fe}=2.42$ apfu. Given the minor modal contents of $\mathrm{K}$-feldspar, biotite is the main potassium-bearing phase. Biotite flakes are often deformed, showing locally kink-band shear domains.

\section{Ilmenite}

Ilmenite forms subhedral, platy crystals 0.2 to $0.5 \mathrm{~mm}$ long, sometimes with imperfect skeletal shapes. The chemical analysis (Table 2) indicates the presence of 7.5 mol. $\% \mathrm{Fe}_{2} \mathrm{O}_{3}$ in solid solution; no unmixed haematite has been noticed in the BSE images. Chromium contents are high, ranging between 0.2 and 0.4 wt. $\% \mathrm{Cr}_{2} \mathrm{O}_{3}$. Slightly elevated $\mathrm{MgO}$ corresponds to $1.4 \mathrm{~mol}$ \% of the geikielite molecule. Surprisingly, tiny and rare lamellae of $\mathrm{ZrO}_{2}$ (probably baddeleyite), up to $3 \mu \mathrm{m}$ long, were noted in the ilmenite. They suggest possible minor isomorphic substitution of $\mathrm{Zr}$ in the ilmenite lattice during the early stage of magma crystallization, before the zircon nucleation commenced. The presence of ilmenite, together with absence of magnetite, indicates relatively reducing conditions of crystallization. 

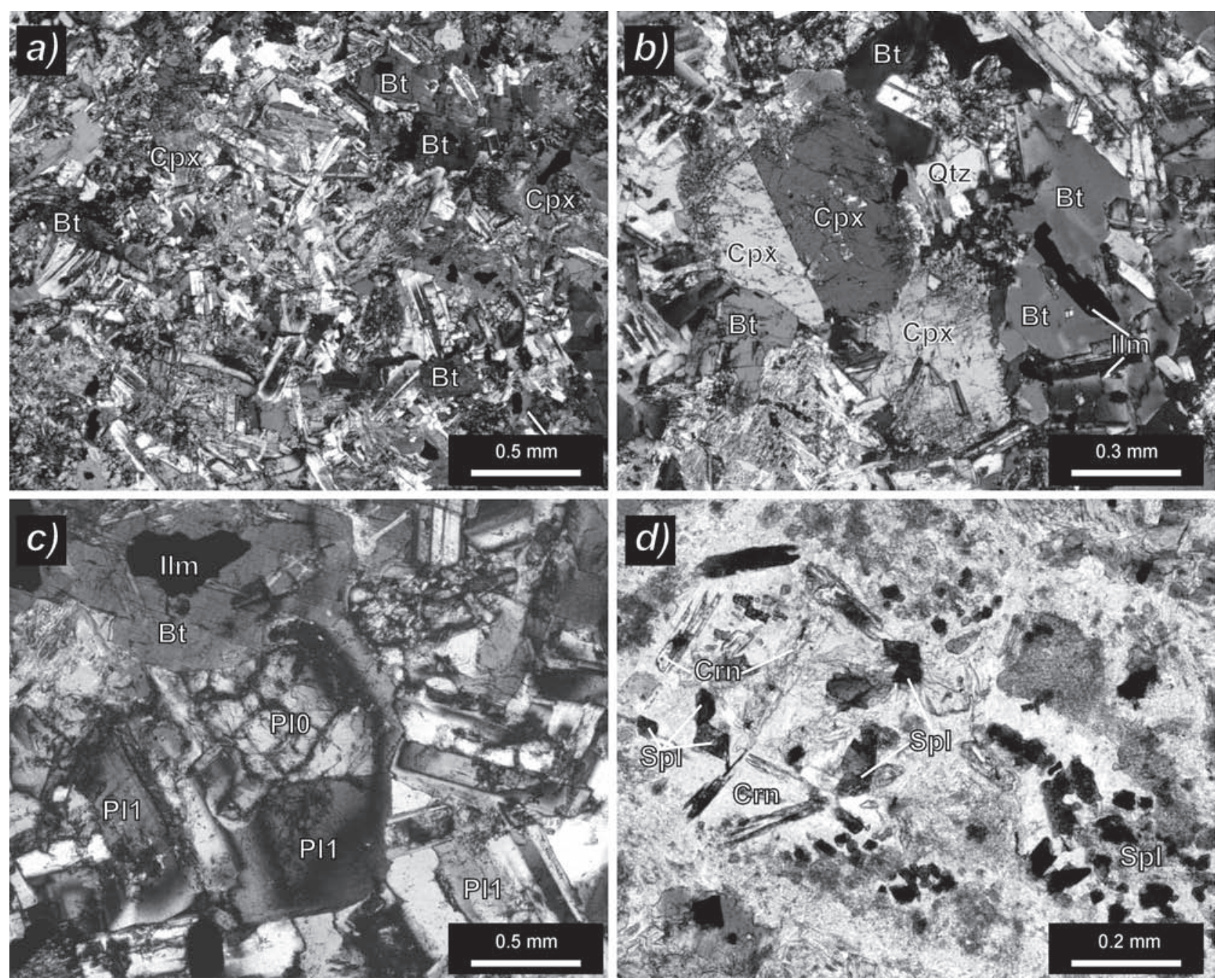

Fig. 3 Photomicrographs of the quartz monzodiorite BR 364 (a-c) and peraluminous enclave BR 367 (d). a - Typical subophitic texture shown by the quartz monzodiorite; $\mathrm{b}$ - Relations of clinopyroxene (Cpx), biotite (Bt), ilmenite (Ilm) and plagioclase (Pl); $\mathrm{c}-\mathrm{Inclusion}$ of a fractured calcic plagioclase (P10) in a zoned plagioclase (P11); d - Al-rich enclave with hercynite (Spl), corundum (Crn) in altered matrix rich in muscovite and plagioclase.

Zircon, baddeleyite and apatite

Zircon was first detected during microprobe work as it occurs in skeletal crystals less than $15 \mu \mathrm{m}$ in size. Figure $4 \mathrm{a}-\mathrm{b}$ shows some of the characteristic shapes of the skeletal zircon crystals, including sceptre terminations and cross-like intergrowths, possibly twinning. Another zirconium phase is $\mathrm{ZrO}_{2}$ in slender prismatic crystals up to $40 \mu \mathrm{m}$ long, interpreted as a probable baddeleyite (Fig. 4d). This mineral is much less abundant than zircon. The crystallization of baddeleyite instead of zircon may reflect locally low activity of silica, adjacent to the crystallizing plagioclase and/or pyroxene. As proposed by Bacon (1989), non-equilibrium concentration gradients may form next to rapidly growing rock-forming minerals, permitting local crystallization of accessories, even when the bulk chemistry of the magma would rule this theoretically out. Apatite occurs in relatively abundant acicular crystals with a length:width ratio near 10 . Hollow distal parts of the crystals are rare.

\section{Other accessory minerals}

Allanite occurs as anhedral grains up to $0.15 \mathrm{~mm}$ long with a weak brown red pleochroism. This mineral is fresh, showing no signs of metamictization. Scarce monazite, not closely associated with allanite, was identified as anhedral grains reaching $20 \mu \mathrm{m}$. Another rare mineral is a probable carbonate-fluoride of REE, which crystallized probably late. The sulphides listed in Table 1 occur mostly as scattered anhedral grains, typically less than $0.2 \mathrm{~mm}$ in size. 
Table 3 Major-element composition of the studied quartz monzodiorites.

\begin{tabular}{|c|c|c|c|c|c|c|c|c|c|c|}
\hline $\begin{array}{l}\text { Sample } \\
\text { Locality }^{3}\end{array}$ & $\begin{array}{r}\text { BR } 193 \\
\text { Hamr }\end{array}$ & $\begin{array}{r}\text { BR } 187 \\
\text { Čítov }\end{array}$ & $\begin{array}{r}\text { BR } 194 \\
\text { Hamr }\end{array}$ & $\begin{array}{l}\text { BR } 185 \\
\text { Skalice }\end{array}$ & $\begin{array}{r}\text { BR } 364 \\
\text { Vlkovice }\end{array}$ & $\begin{array}{r}\text { Li-2 } \\
\text { Skalice }\end{array}$ & $\begin{array}{r}\text { Li-1 } \\
\text { Skalice }\end{array}$ & $\begin{array}{l}\text { BR } 369 \\
\text { Skalice }\end{array}$ & $\begin{array}{r}\text { BR } \text { 188 }^{1} \\
\text { Čítov }\end{array}$ & $\begin{array}{c}\text { BR 359² } \\
\text { Skalice }\end{array}$ \\
\hline $\mathrm{SiO}_{2}$ & 51.77 & 51.06 & 51.16 & 51.50 & 50.54 & 51.88 & 51.73 & 52.60 & 53.42 & 73.50 \\
\hline $\mathrm{TiO}_{2}$ & 1.46 & 1.49 & 1.59 & 1.59 & 1.57 & 1.48 & 1.48 & 1.49 & 1.53 & 0.05 \\
\hline $\mathrm{Al}_{2} \mathrm{O}_{3}$ & 15.71 & 15.69 & 15.78 & 15.64 & 16.04 & 15.93 & 16.01 & 15.90 & 15.83 & 14.21 \\
\hline $\mathrm{Fe}_{2} \mathrm{O}_{3}$ & 1.03 & 0.75 & 0.98 & 1.03 & 0.67 & 0.99 & 1.03 & 0.98 & 0.67 & 0.28 \\
\hline $\mathrm{FeO}$ & 7.46 & 7.67 & 7.66 & 7.53 & 7.78 & 6.85 & 7.06 & 6.92 & 7.52 & 0.39 \\
\hline MnO & 0.150 & 0.140 & 0.150 & 0.140 & 0.140 & 0.124 & 0.126 & 0.130 & 0.150 & 0.03 \\
\hline MgO & 7.61 & 6.92 & 6.80 & 6.73 & 6.46 & 6.45 & 6.19 & 5.71 & 5.18 & 0.13 \\
\hline $\mathrm{CaO}$ & 6.37 & 6.82 & 7.11 & 6.96 & 7.35 & 6.81 & 6.87 & 6.83 & 6.58 & 0.61 \\
\hline $\mathrm{Li}_{2} \mathrm{O}$ & 0.006 & 0.006 & 0.009 & 0.004 & 0.004 & 0.005 & 0.006 & 0.005 & 0.005 & $<0.001$ \\
\hline $\mathrm{Na}_{2} \mathrm{O}$ & 2.57 & 2.73 & 2.78 & 2.52 & 2.62 & 2.39 & 2.42 & 2.55 & 3.06 & 3.99 \\
\hline $\mathbf{K}_{2} \mathbf{O}$ & 2.37 & 2.28 & 2.35 & 2.39 & 2.48 & 2.52 & 2.85 & 2.64 & 2.43 & 5.13 \\
\hline $\mathrm{P}_{2} \mathrm{O}_{5}$ & 0.38 & 0.34 & 0.36 & 0.44 & 0.39 & 0.485 & 0.487 & 0.42 & 0.39 & 0.33 \\
\hline $\mathrm{CO}_{2}$ & 0.18 & 0.03 & 0.07 & 0.10 & 0.19 & 0.07 & 0.08 & 0.08 & 0.26 & 0.02 \\
\hline C & 0.03 & 0.04 & 0.02 & 0.02 & 0.03 & 0.04 & 0.03 & 0.04 & 0.02 & 0.02 \\
\hline $\mathrm{H}_{2} \mathrm{O}+$ & 2.77 & 2.82 & 2.70 & 2.95 & 2.60 & 3.12 & 3.19 & 2.64 & 2.43 & 0.86 \\
\hline $\mathbf{F}$ & 0.10 & 0.05 & 0.06 & 0.10 & 0.09 & 0.119 & 0.122 & 0.05 & 0.08 & 0.03 \\
\hline $\mathbf{S}$ & 0.12 & 0.14 & 0.03 & 0.22 & 0.13 & 0.090 & 0.100 & 0.09 & 0.43 & 0.02 \\
\hline $\mathrm{H}_{2} \mathrm{O}-$ & 0.12 & 0.13 & 0.11 & 0.10 & 0.13 & 0.23 & 0.19 & 0.14 & 0.14 & 0.13 \\
\hline F-ekv & -0.04 & -0.02 & -0.03 & -0.04 & -0.04 & -0.050 & -0.051 & -0.02 & -0.03 & 0.01 \\
\hline S-ekv & -0.03 & -0.04 & $-<0.01$ & -0.06 & -0.03 & -0.022 & -0.025 & -0.02 & -0.11 & $-<0.01$ \\
\hline Sum & 100.13 & 99.04 & 99.68 & 99.85 & 99.14 & 99.51 & 99.89 & 99.18 & 99.99 & 99.71 \\
\hline mg\# & 61.8 & 59.6 & 58.7 & 58.7 & 57.9 & 59.8 & 58.0 & 56.6 & 53.2 & 26.5 \\
\hline A/CNK & 0.9 & 0.8 & 0.8 & 0.8 & 0.8 & 0.8 & 0.8 & 0.8 & 0.8 & 1.1 \\
\hline A/NK & 2.3 & 2.3 & 2.2 & 2.3 & 2.3 & 2.4 & 2.3 & 2.3 & 2.1 & 1.2 \\
\hline $\mathrm{Na}_{2} \mathrm{O} / \mathrm{K}_{2} \mathrm{O}$ & 1.1 & 1.2 & 1.2 & 1.1 & 1.1 & 0.9 & 0.8 & 1.0 & 1.3 & 0.8 \\
\hline \multicolumn{11}{|c|}{ CIPW normative composition (CIPW norm with biotite and hornblende, Hutchison 1975) } \\
\hline Q & 9.17 & 7.16 & 6.90 & 9.65 & 7.47 & 11.77 & 11.74 & 12.57 & 11.80 & 30.34 \\
\hline $\mathrm{C}$ & 0.00 & 0.00 & 0.00 & 0.00 & 0.00 & 0.00 & 0.00 & 0.00 & 0.00 & 1.82 \\
\hline Or & 0.00 & 0.00 & 0.00 & 0.00 & 0.00 & 0.00 & 0.00 & 0.00 & 0.00 & 29.72 \\
\hline $\mathbf{A b}$ & 21.75 & 23.10 & 23.52 & 21.32 & 22.17 & 20.22 & 20.48 & 21.58 & 25.89 & 33.76 \\
\hline An & 24.33 & 23.82 & 23.64 & 24.30 & 24.68 & 25.29 & 24.40 & 24.14 & 22.28 & 0.74 \\
\hline Wo & 0.00 & 0.00 & 0.00 & 0.00 & 0.00 & 0.00 & 0.17 & 0.56 & 0.09 & 0.00 \\
\hline Mt & 1.49 & 1.09 & 1.42 & 1.49 & 0.97 & 1.44 & 1.49 & 1.42 & 0.97 & 0.41 \\
\hline II & 2.77 & 2.83 & 3.02 & 3.02 & 2.98 & 2.81 & 2.81 & 2.83 & 2.91 & 0.10 \\
\hline Ap & 0.90 & 0.81 & 0.85 & 1.04 & 0.92 & 1.15 & 1.13 & 0.99 & 0.92 & 0.78 \\
\hline $\mathbf{F r}$ & 0.14 & 0.04 & 0.06 & 0.12 & 0.11 & 0.16 & 0.16 & 0.03 & 0.09 & 0.00 \\
\hline Pr & 0.22 & 0.26 & 0.06 & 0.41 & 0.24 & 0.17 & 0.19 & 0.17 & 0.80 & 0.04 \\
\hline $\mathrm{Cc}$ & 0.41 & 0.07 & 0.16 & 0.23 & 0.43 & 0.16 & 0.18 & 0.18 & 0.59 & 0.05 \\
\hline $\mathbf{B i}$ & 21.50 & 20.82 & 21.47 & 21.78 & 22.74 & 22.92 & 26.01 & 24.17 & 22.42 & 0.95 \\
\hline Но & 4.79 & 11.34 & 13.13 & 9.52 & 11.64 & 6.55 & 7.70 & 7.71 & 8.61 & 0.00 \\
\hline Sum & 87.47 & 91.33 & 94.23 & 92.89 & 94.36 & 92.64 & 96.47 & 96.35 & 97.39 & 98.70 \\
\hline
\end{tabular}

(Samples are arranged in the sequence of decreasing $\mathrm{MgO}$ )

${ }^{1}$ Sample BR 188 contains partly assimilated enclaves

${ }^{2}$ BR 359 is a leucogranite xenolith enclosed in the quartz monzodiorite

${ }^{3}$ For localities, see Fig. 1b-c 

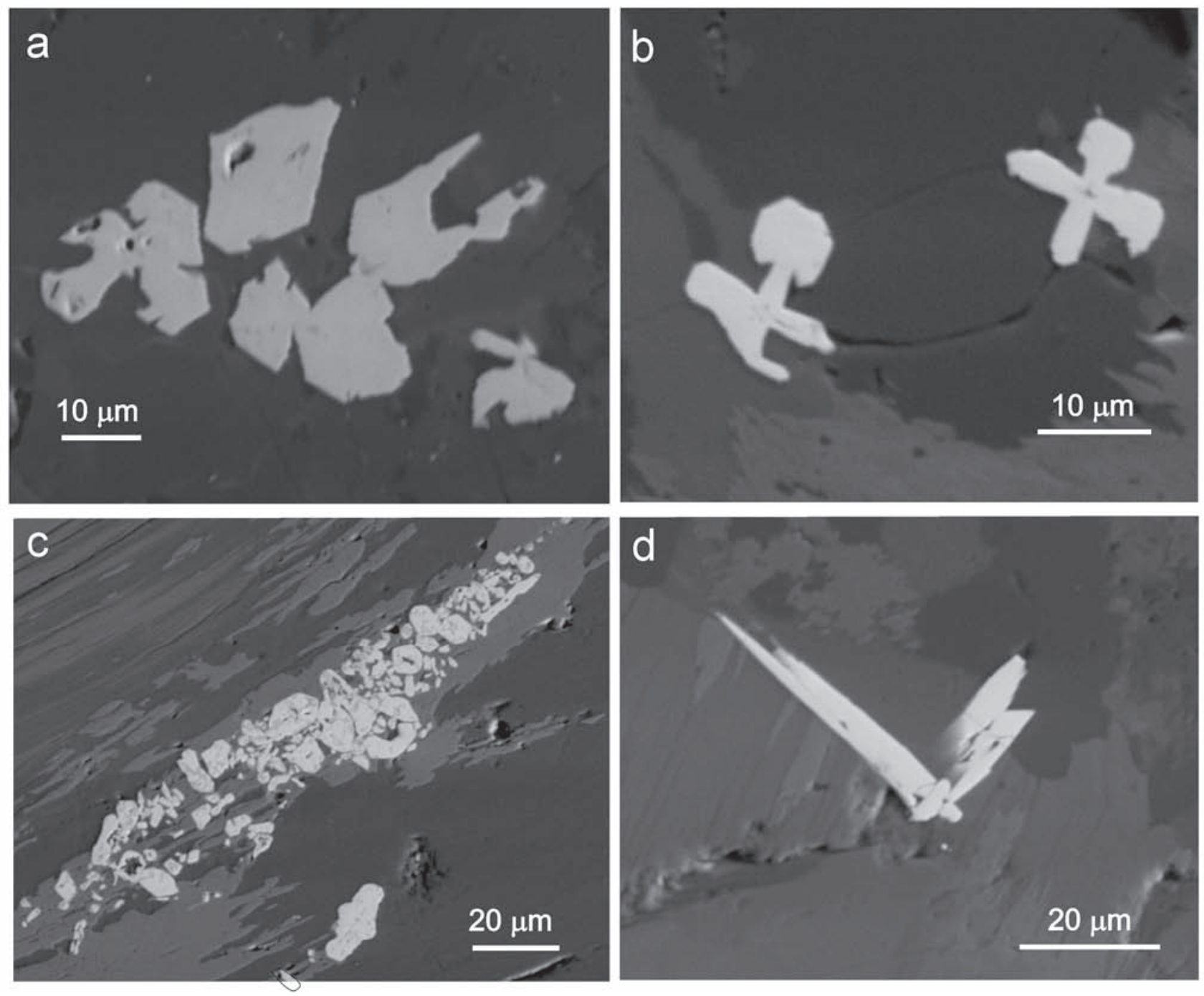

Fig. 4 Crystal morphology of the Zr minerals. a - Skeletal zircon grains; b - Intergrowth (twinning?) of zircon crystals with sceptre termination; $\mathrm{c}$ - Aggregate of minute euhedral zircon crystals; $\mathrm{d}$ - Prismatic crystals of baddeleyite.

Petrology and mineralogy of the Al-rich inclusions

The Al-rich enclaves, up to $3 \mathrm{~mm}$ long, usually embrace newly formed corundum and hercynitic spinel set in a fine-grained plagioclase and muscovite (sericite) matrix (Fig. 3d). Spinel contains $\mathrm{Fe}^{2+}=0.522$ with $\mathrm{Mg}=0.466$ apfu and only $1.8 \mathrm{~mol}$ \% gahnite. Some of the enclaves contain at least relict structures after sheaves of fibrolitic sillimanite. It is possible that the sericite aggregates formed by alteration of cordierite. There are examples of small subhedral plagioclase crystals, arranged radially around Al-rich enclaves.

\section{Whole-rock geochemistry}

Table 3 summarises newly obtained major- and minorelement whole-rock geochemical data for nine samples of dykes and one leucogranitic xenolith (BR 359). The samples come from the quarries Skalice and Vlkovice, as well as loose blocks in the Hamr village and the Čí-

Fig. 5 a - Multicationic P-Q plot (Debon - Le Fort 1983, 1988). $P$ represents the proportion of K-feldspar to plagioclase and $Q$ the quartz content; $\mathrm{b}-$ Diagram $\mathrm{SiO}_{2}-\left(\mathrm{Na}_{2} \mathrm{O}+\mathrm{K}_{2} \mathrm{O}\right)(\mathrm{TAS})$ of Cox et al. (1979). The dividing line between the subalkaline and alkaline domains is after Irvine - Baragar (1971); c - Ternary plot $\mathrm{Na}_{2} \mathrm{O}-\mathrm{Al}_{2} \mathrm{O}_{3}-\mathrm{K}_{2} \mathrm{O}$ (mol. \%). Dashed lines define the following compositional fields: peraluminous/metaluminous, $\left(\mathrm{Na}_{2} \mathrm{O}+\mathrm{K}_{2} \mathrm{O}\right) / \mathrm{Al}_{2} \mathrm{O}_{3}<1$; peralkaline, $\left(\mathrm{Na}_{2} \mathrm{O}+\mathrm{K}_{2} \mathrm{O}\right) / \mathrm{Al}_{2} \mathrm{O}_{3}>1$; perpotassic, $\mathrm{K}_{2} \mathrm{O} / \mathrm{Al}_{2} \mathrm{O}_{3}>1$; potassic, $1<\mathrm{K} 2 \mathrm{O} / \mathrm{Na}_{2} \mathrm{O}<3$; and ultrapotassic, $\mathrm{K}_{2} \mathrm{O} / \mathrm{Na}_{2} \mathrm{O}=3$ in mol. $\%$ ( or $\mathrm{K}_{2} \mathrm{O} / \mathrm{Na}_{2} \mathrm{O}=2$ in wt. \%, which is equivalent to the definition of ultrapotassic igneous rocks by Foley et al. 1987 ; $\mathrm{d}-\mathrm{AFM}$ diagram $\left(\mathrm{A}=\mathrm{Na}_{2} \mathrm{O}+\mathrm{K}_{2} \mathrm{O}, \mathrm{F}=\mathrm{FeOt}, \mathrm{M}=\mathrm{MgO}\right.$ : Irvine - Baragar 1971) illustrating the calc-alkaline trend shown by the analysed samples; e - $\mathrm{SiO}_{2}-\mathrm{K}_{2} \mathrm{O}$ plot with the discrimination boundaries between the tholeiitic, calc-alkaline, high-K calc-alkaline and shoshonitic rocks of Peccerillo - Taylor (1976). Compositions of the dyke rocks from Ševětín (Vrána et al. 1993) and Sušice (Vrána 2004) swarms are shown for comparison. 


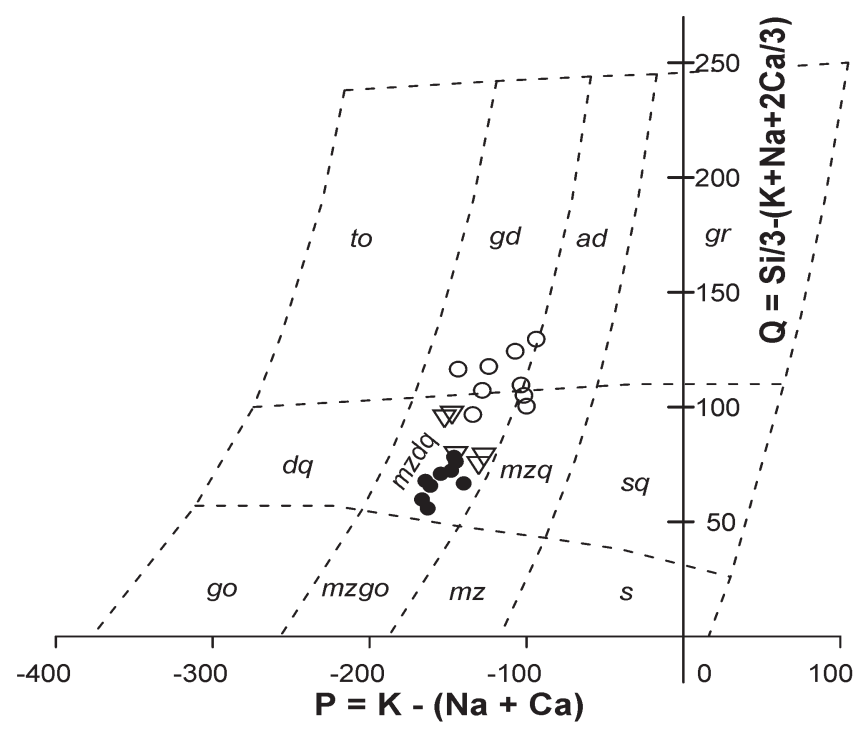

a)

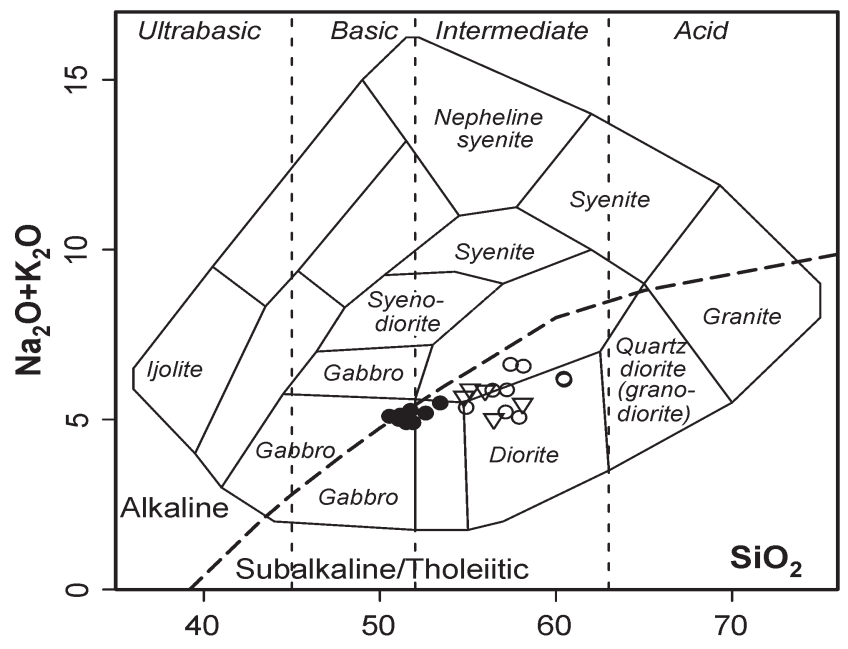

b)

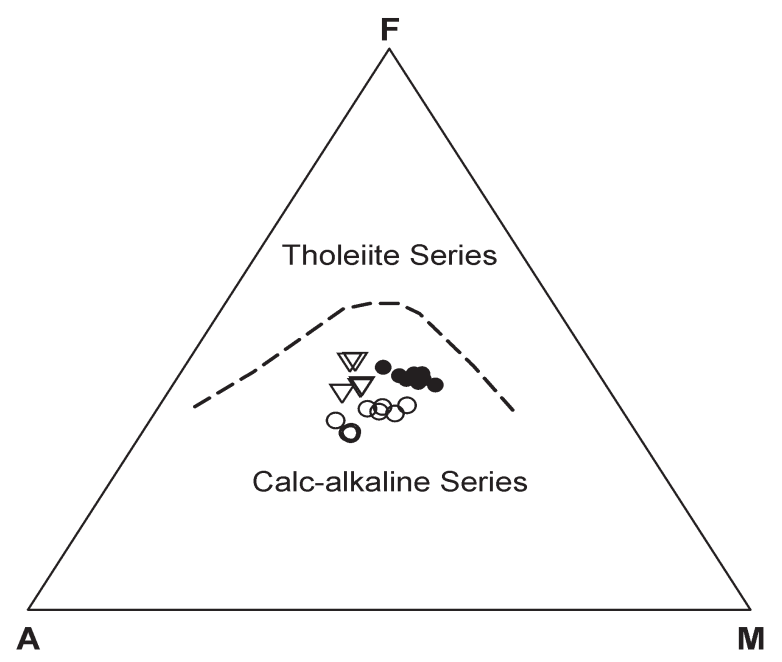

\section{)}

ad: adamellite

dq: quartz diorite, quartz gabbro

quartz anorthosite

gd: granodiorite

go: gabbro, diorite, anorthosite

gr: granite

mz: monzonite

mzdq: quartz monzodiorite, quartz monzogabbro

mzgo: monzogabbro,

mzq: quartz monzonite

s: $\quad$ syenite

sq: quartz syenite

to: $\quad$ tonalite, trondhjemite

Štěpánovice dykes

Ševětín dyke swarm

$\nabla$ Sušice dyke swarm
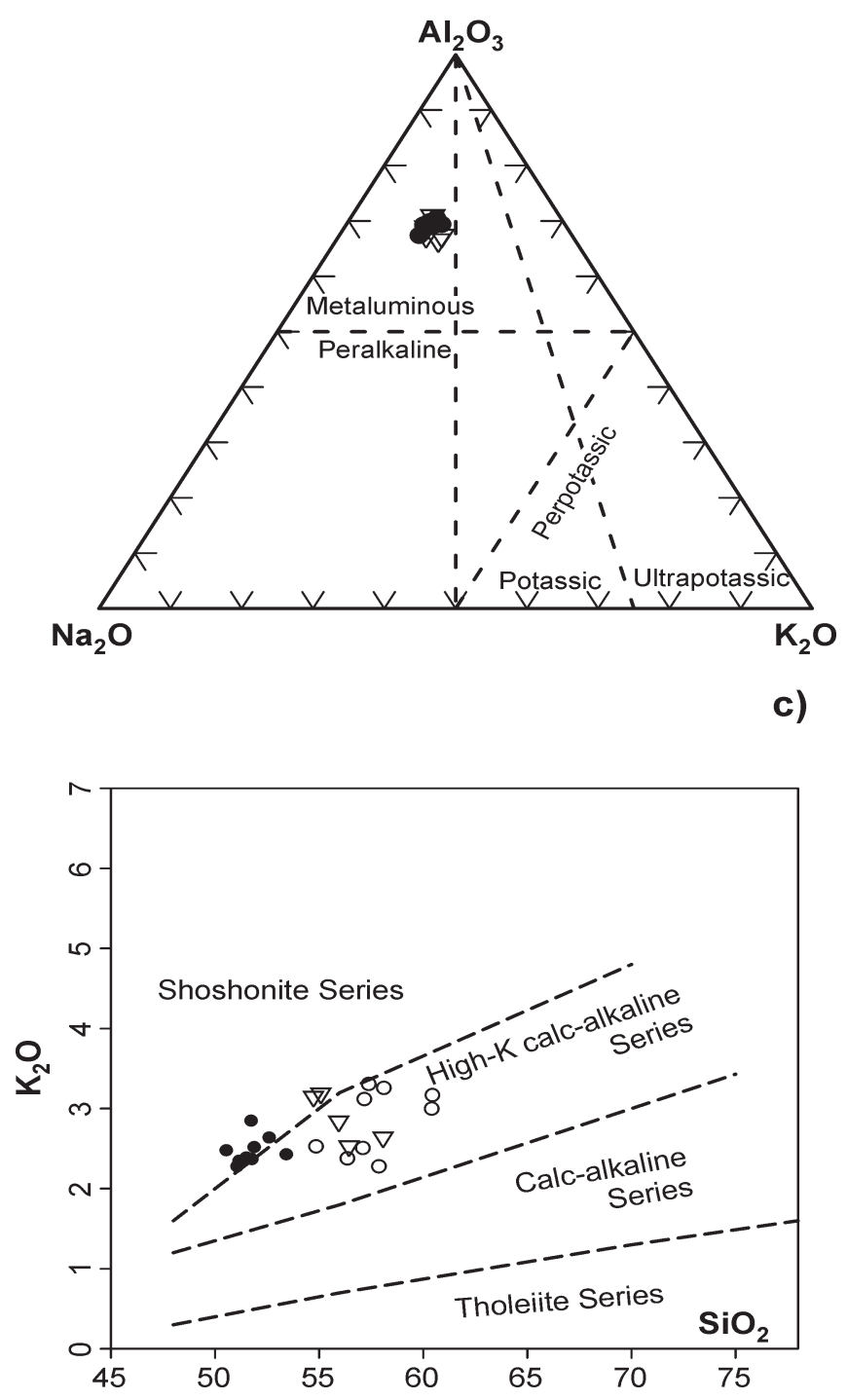

d) 
tov forest (see Figs 1b-c for the location of the sampling sites).

\section{Major and minor elements}

The major-element composition of the studied rocks shows only a limited intrasuite variation. All rocks correspond to quartz monzodiorites in the multicationic $\mathrm{P}$ Q diagram of Debon - Le Fort (1983, 1988) (Fig. 5a). The mg-numbers $(m g \#=$ molar $\mathrm{Mg} /(\mathrm{Mg}+\mathrm{Fet}))$ are rather high, changing a little between 53 and 62 . The dykes are basic-intermediate (50.54-53.42 wt. \% $\mathrm{SiO}_{2}$; the most siliceous being the contaminated sample BR 188), with the content of alkalis close to, but mostly below, the dividing line between the subalkaline and alkaline rocks after Irvine - Baragar (1971, Fig. 5b). In the TAS diagram of Cox et al. (1979), which unfortunately does not take into account monzonitic compositions, the samples would classify as gabbros/gabbrodiorites.

The studied mafic dykes have $\mathrm{K}_{2} \mathrm{O} / \mathrm{Na}_{2} \mathrm{O}$ ratios close to unity $(0.79-1.18$, wt. \%). In terms of the molar proportions, $\mathrm{Na}$ prevails over $\mathrm{K}$ in all samples, as demon-

Table 4 Trace-element composition of the studied quartz monzodiorites (ppm).

\begin{tabular}{|c|c|c|c|c|c|c|c|c|c|c|}
\hline Sample & $\begin{array}{l}\text { BR } 193 \\
\text { Hamr }\end{array}$ & $\begin{array}{c}\text { BR } 187 \\
\text { Čítov }\end{array}$ & $\begin{array}{l}\text { BR } 194 \\
\text { Hamr }\end{array}$ & $\begin{array}{l}\text { BR } 185 \\
\text { Skalice }\end{array}$ & $\begin{array}{r}\text { BR } 364 \\
\text { Vlkovice }\end{array}$ & $\begin{array}{c}\text { Li-2 } \\
\text { Skalice }\end{array}$ & $\begin{array}{c}\text { Li-1 } \\
\text { Skalice }\end{array}$ & $\begin{array}{l}\text { BR } 369 \\
\text { Skalice }\end{array}$ & $\begin{array}{c}\text { BR } 188 \\
\text { Čítov }\end{array}$ & $\begin{array}{l}\text { BR } 359 \\
\text { Skalice }\end{array}$ \\
\hline $\mathbf{R b}$ & 68 & 64 & 73 & 63 & 70 & 77 & 84 & 72 & 66 & 191 \\
\hline Cs & 5.0 & 5.7 & 4.3 & 2.3 & 2.5 & 1.8 & 1.5 & 2.1 & 5.1 & 7.6 \\
\hline $\mathbf{B a}$ & 1635 & 1684 & 1398 & 1606 & 1447 & 2125 & 2321 & 1958 & 1706 & 237 \\
\hline $\mathrm{Sr}$ & 871 & 868 & 803 & 796 & 870 & 975 & 992 & 1083 & 865 & 87 \\
\hline $\mathbf{Z r}$ & 246 & 227 & 228 & 263 & 240 & 273 & 276 & 279 & 270 & 23 \\
\hline Hf & 6.6 & 5.9 & 5.9 & 6.7 & 5.8 & 7.1 & 7.6 & 7.1 & 7.0 & 1.6 \\
\hline $\mathrm{Nb}$ & 13.3 & 12.0 & 13.2 & 14.9 & 13.8 & 14.8 & 14.9 & 15.2 & 14.8 & 18.8 \\
\hline Ta & 0.8 & 0.8 & 0.7 & 0.9 & 0.8 & 0.9 & 0.8 & 0.9 & 0.9 & 5.9 \\
\hline Ga & 21 & 20 & 21 & 19 & 21 & 22 & 23 & 21 & 23 & 18 \\
\hline Th & 10.1 & 10.2 & 9.4 & 12.1 & 9.8 & 14.9 & 15.1 & 14.6 & 12.0 & 0.4 \\
\hline $\mathbf{U}$ & 2.3 & 2.3 & 2.4 & 2.6 & 2.4 & 3.0 & 3.0 & 3.1 & 2.6 & 2.0 \\
\hline Co & 36 & 35 & 33 & 32 & 35 & 30 & 31 & 29 & 31 & 5 \\
\hline $\mathrm{Ni}$ & - & - & - & - & - & 57 & 57 & - & - & - \\
\hline $\mathbf{V}$ & 139 & 146 & 149 & 144 & 138 & 141 & 147 & 139 & 140 & 9 \\
\hline $\mathrm{Zn}$ & 68 & 64 & 76 & 69 & 72 & 68 & 72 & 71 & 64 & 15 \\
\hline W & 0.7 & 0.5 & 0.8 & 0.5 & 0.6 & 0.4 & 0.4 & 0.6 & 2.4 & 1.3 \\
\hline $\mathrm{Cu}$ & 26 & 25 & 15 & 23 & 15 & 21 & 24 & 25 & 20 & 8 \\
\hline $\mathbf{P b}$ & 47.0 & 15.3 & 8.3 & 13.9 & 9.0 & 15.8 & 21.4 & 16.0 & 7.5 & 7.1 \\
\hline As & 7.1 & 6.0 & 6.8 & 0.9 & 3.6 & 8.1 & 6.5 & 3.5 & 1.1 & 0.6 \\
\hline La & 78.7 & 77.4 & 63.7 & 78.8 & 73.4 & 100.9 & 101.6 & 103.6 & 87.1 & 4.4 \\
\hline $\mathrm{Ce}$ & 157.5 & 157.1 & 130.8 & 159.4 & 151.3 & 207.8 & 210.2 & 215.8 & 176.7 & 6.1 \\
\hline Pr & 19.05 & 19.16 & 16.40 & 19.57 & 18.58 & 24.91 & 25.23 & 25.93 & 20.93 & 0.84 \\
\hline Nd & 72.5 & 70.0 & 61.3 & 74.6 & 70.7 & 95.7 & 99.8 & 99.8 & 77.8 & 2.7 \\
\hline $\mathrm{Sm}$ & 11.2 & 10.3 & 9.9 & 11.6 & 10.6 & 13.9 & 14.0 & 13.9 & 11.8 & 0.7 \\
\hline Eu & 2.92 & 2.72 & 2.51 & 2.84 & 2.78 & 3.22 & 3.36 & 3.41 & 3.17 & 0.18 \\
\hline Gd & 7.57 & 6.77 & 6.67 & 7.58 & 7.17 & 8.64 & 8.84 & 8.61 & 7.81 & 0.79 \\
\hline $\mathbf{T b}$ & 1.14 & 1.05 & 1.08 & 1.16 & 1.07 & 1.27 & 1.29 & 1.16 & 1.18 & 0.17 \\
\hline Dy & 4.97 & 4.93 & 4.81 & 5.51 & 5.16 & 5.38 & 5.97 & 5.81 & 5.71 & 1.03 \\
\hline Но & 0.93 & 0.91 & 0.91 & 0.99 & 0.92 & 0.97 & 1.01 & 1.01 & 1.02 & 0.19 \\
\hline $\mathbf{E r}$ & 2.74 & 2.65 & 2.81 & 2.95 & 2.89 & 2.73 & 2.93 & 3.02 & 3.16 & 0.62 \\
\hline $\mathbf{T m}$ & 0.43 & 0.40 & 0.36 & 0.44 & 0.42 & 0.43 & 0.45 & 0.44 & 0.46 & 0.10 \\
\hline $\mathbf{Y b}$ & 2.52 & 2.35 & 2.46 & 2.61 & 2.55 & 2.54 & 2.65 & 2.80 & 2.84 & 0.68 \\
\hline Lu & 0.35 & 0.34 & 0.35 & 0.38 & 0.36 & 0.36 & 0.37 & 0.39 & 0.42 & 0.12 \\
\hline $\mathbf{Y}$ & 27.5 & 27.4 & 27.5 & 29.6 & 26.9 & 29.9 & 30.6 & 31.2 & 31.4 & 7.4 \\
\hline $\mathbf{E u} / \mathbf{E u} *$ & 0.97 & 1.00 & 0.94 & 0.93 & 0.98 & 0.90 & 0.92 & 0.95 & 1.01 & 0.74 \\
\hline $\mathbf{L} \mathbf{a}_{\mathrm{N}} / \mathbf{Y} \mathbf{b}_{\mathrm{N}}$ & 21.06 & 22.21 & 17.46 & 20.35 & 19.41 & 26.78 & 25.85 & 24.95 & 20.68 & 4.36 \\
\hline $\mathbf{L a}_{N} / \mathrm{Sm}_{\mathrm{N}}$ & 4.42 & 4.73 & 4.05 & 4.27 & 4.36 & 4.57 & 4.56 & 4.69 & 4.64 & 3.95 \\
\hline इREE & 362.52 & 356.08 & 304.06 & 368.43 & 347.90 & 468.75 & 477.70 & 485.68 & 400.10 & 18.62 \\
\hline
\end{tabular}


Table $5 \mathrm{Sr}-\mathrm{Nd}$ isotopic data for the studied quartz monzodiorites.

\begin{tabular}{|c|c|c|c|c|c|c|c|c|c|c|c|c|c|c|c|}
\hline Sample & $\begin{array}{c}\mathbf{R b} \\
(\mathbf{p p m})\end{array}$ & $\begin{array}{c}\mathrm{Sr} \\
(\mathbf{p p m})\end{array}$ & ${ }^{87} \mathrm{Rb} /{ }^{86} \mathrm{Sr}$ & ${ }^{87} \mathrm{Sr} /{ }^{86} \mathrm{Sr}$ & 2 s.e. & $\left({ }^{87} \mathrm{Sr} /{ }^{86} \mathrm{Sr}\right)_{\mathrm{i}}$ & $\begin{array}{c}\text { Sm } \\
(\mathbf{p p m})\end{array}$ & $\begin{array}{c}\text { Nd } \\
(\mathbf{p p m})\end{array}$ & ${ }^{147} \mathrm{Sm} /{ }^{144} \mathrm{Nd}$ & ${ }^{143} \mathrm{Nd} /{ }^{144} \mathrm{Nd}$ & 2 s.e & $\left({ }^{143} \mathrm{Nd} /{ }^{144} \mathrm{Nd}\right)_{\mathrm{i}}$ & $\varepsilon_{\mathrm{Nd}}^{\mathrm{i}}$ & $\begin{array}{l}T_{\text {CHUR }}^{\mathrm{Nd}} \\
\text { (Ga) }\end{array}$ & $\begin{array}{l}\mathrm{T}_{\mathrm{DM}}^{\mathrm{Nd}} \\
(\mathrm{Ga})\end{array}$ \\
\hline Li-1 & 84.4 & 991.6 & 0.2463 & 0.707404 & 32 & 0.70639 & 14.0 & 99.8 & 0.0848 & 0.512293 & 4 & 0.512132 & -2.6 & 0.47 & 0.97 \\
\hline Li-2 & 77.3 & 975.2 & 0.2293 & 0.707533 & 16 & 0.70659 & 13.9 & 95.7 & 0.0878 & 0.512272 & 10 & 0.512105 & -3.1 & 0.51 & 1.02 \\
\hline
\end{tabular}

Isotopic ratios with subscript 'i' were all age-corrected to $290 \mathrm{Ma}$

$\left(\mathrm{T}_{\mathrm{DM}}^{\mathrm{Nd}}\right)=$ single-stage Nd model ages calculated after Liew \& Hofmann (1988).

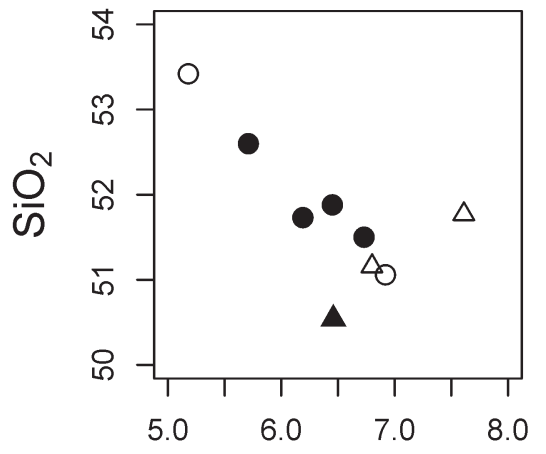

$\mathrm{MgO}$

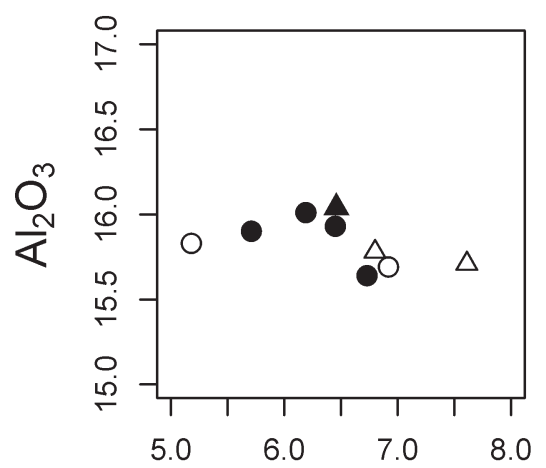

$\mathrm{MgO}$

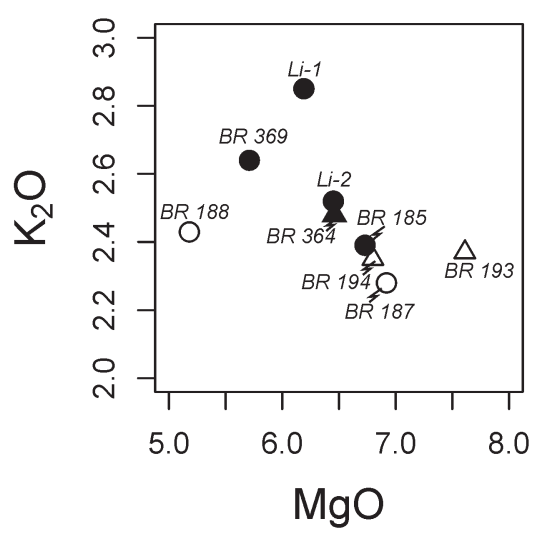

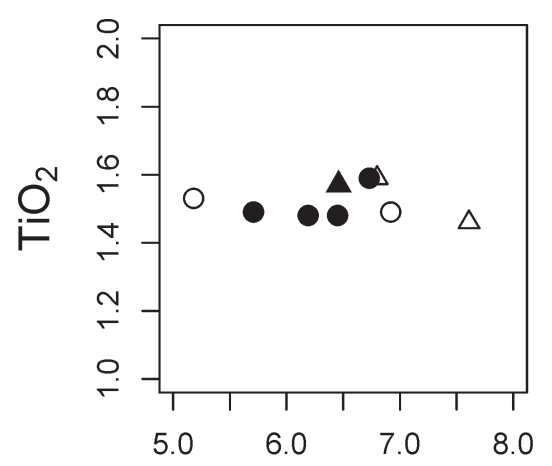

$\mathrm{MgO}$
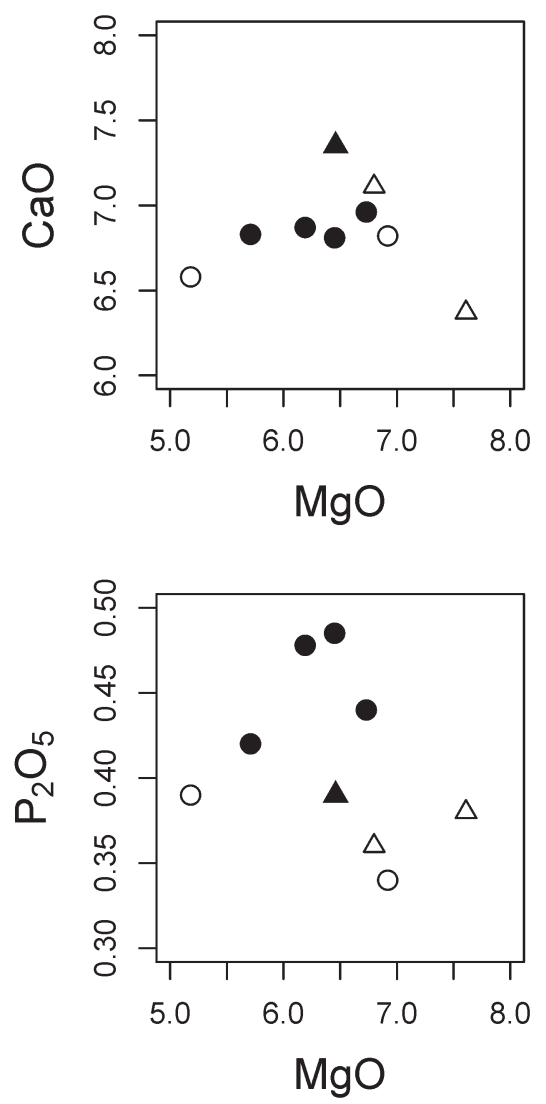
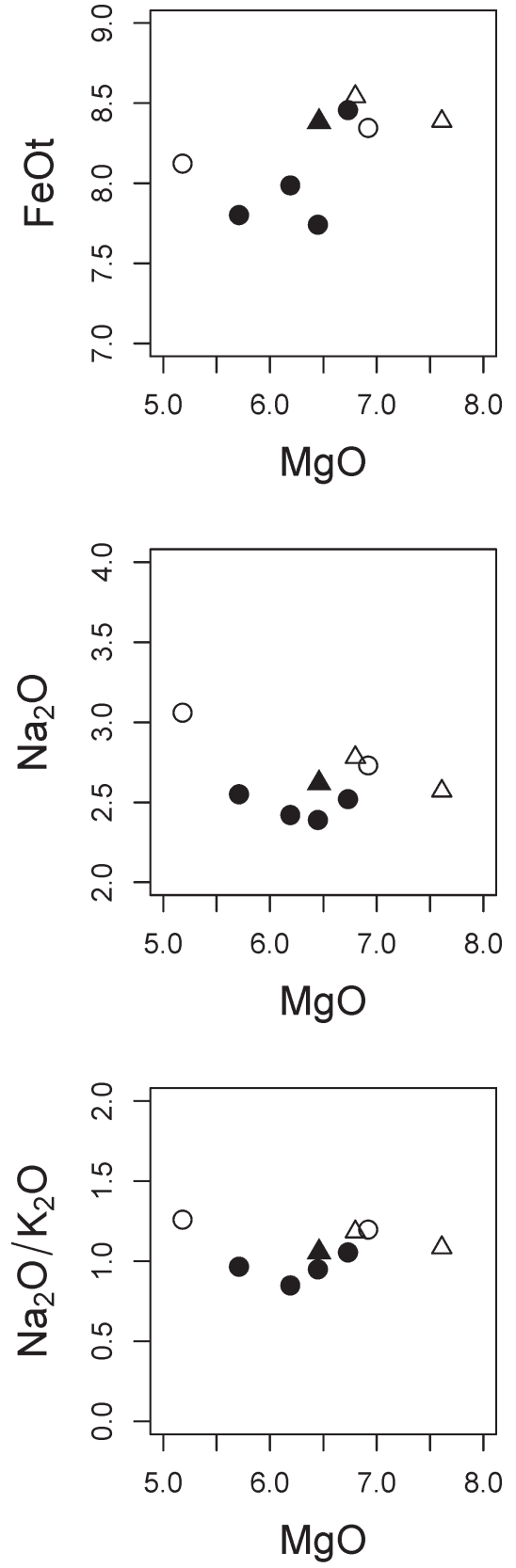

Skalice $\Delta$ Vlkovice OČítov $\Delta$ Hamr

Fig. 6 Binary plots of $\mathrm{MgO}$ versus major- and minor elements for the studied dykes. 
strated by the ternary diagram $\mathrm{Na}_{2} \mathrm{O}-\mathrm{Al}_{2} \mathrm{O}_{3}-\mathrm{K}_{2} \mathrm{O}$ (Fig. 5c). All samples are metaluminous, with the Shand index, $\mathrm{A} / \mathrm{CNK}=$ molar $\mathrm{Al}_{2} \mathrm{O}_{3} /\left(\mathrm{CaO}+\mathrm{Na}_{2} \mathrm{O}+\mathrm{K}_{2} \mathrm{O}\right)$, being significantly lower than $1(\mathrm{~A} / \mathrm{CNK}=0.79-0.86)$.

The rocks can be characterized as calc-alkaline (see the AFM ternary of Irvine- Baragar 1971; Fig. 5d). Most of the samples are shoshonitic, judging from the $\mathrm{SiO}_{2}-$ $\mathrm{K}_{2} \mathrm{O}$ diagram (Peccerillo - Taylor 1976; Fig. 5e).

As is often the case with mafic intrusive rocks, the $\mathrm{MgO}$ seems to be a more appropriate fractionation index than silica. The variation is definitely larger (5.2-7.6 wt. \%; the lowest $\mathrm{MgO}$ contents in the contaminated sample BR 188), presumably due to the crystallization of ferromagnesian phases. There is a strong negative correlation between the whole-rock $\mathrm{MgO}$ and $\mathrm{SiO}_{2}$ (Fig. 6). Other major elements show conspicuous convex upward trends with inflection at $\mathrm{MgO} \sim 6.3$ wt. $\%\left(\mathrm{Al}_{2} \mathrm{O}_{3}, \mathrm{~K}_{2} \mathrm{O}, \mathrm{P}_{2} \mathrm{O}_{5}\right.$ and $\mathrm{CaO})$. On the other hand, the plots involving FeOt and $\mathrm{Na}_{2} \mathrm{O}$ fail to define a simple trend; $\mathrm{TiO}_{2}$ contents are remarkably constant throughout the studied suite.

\section{Trace elements}

The trace-element evolution is shown in the form of binary plots with $\mathrm{MgO}$ on abscissa (Fig. 7). Similar to major- and minor element oxides, some, mostly feldsparcompatible elements form convex upward trends $(\mathrm{Rb}, \mathrm{Ba}$ and $\mathrm{Pb}$ ). Other elements behave incompatibly (i.e. their contents increase with increasing degrees of fractionation, expressed by decreasing $\mathrm{MgO}$ ): $\mathrm{Zr}, \mathrm{Nb}, \mathrm{Y}, \mathrm{Th}$ and $\mathrm{U}$. Taking apart the contaminated sample BR 188, there are marked differences between the samples from the Skalice quarry and rest of the dataset. Yttrium in samples from the Skalice quarry is systematically higher than $29 \mathrm{ppm}, \mathrm{Zr}>250 \mathrm{ppm}, \mathrm{Nb}>14 \mathrm{ppm}, \mathrm{Th}>12$ and $\mathrm{U}>2.6$ (Fig. 7, Table 4).

The chondrite-normalized (Boynton 1984) REE patterns tend to be rather uniform (Fig. 8a), with total REE concentrations ranging between 304 and $486 \mathrm{ppm}$. The patterns are all steep, with notable LREE/HREE $\left(\mathrm{La}_{\mathrm{N}} /\right.$ $\left.\mathrm{Yb}_{\mathrm{N}}=17.5-26.8\right)$ and LREE/MREE $\left(\mathrm{La}_{\mathrm{N}} / \mathrm{Sm}_{\mathrm{N}}=4.0-4.7\right)$ enrichments. The Eu anomaly is absent or negligibly negative $\left(\mathrm{Eu} / \mathrm{Eu}^{*}=1.01-0.90\right)$. There is a faint tendency to higher $\Sigma$ REE, $\mathrm{La}_{\mathrm{N}} / \mathrm{Yb}_{\mathrm{N}}$ and $\mathrm{La}_{\mathrm{N}} / \mathrm{Sm}_{\mathrm{N}}$ as well as lower $\mathrm{Eu} /$ $\mathrm{Eu}^{*}$ ratios with the rising silica (Fig. $8 \mathrm{~b}$ ).

The compositions of the quartz monzodiorites were normalized by an average Primitive Mantle (PM: Sun McDonough 1989) (Fig. 8c). The patterns are all enriched in LILE, starting at $>190-722 \times \mathrm{PM}(\mathrm{Cs})$ and falling to slightly less than $5 \times$ PM (Lu). All show superimposed troughs in $\mathrm{Nb}$ and (much less noticeable), Ti. The other striking feature is a variable enrichment in $\mathrm{Pb} ; \mathrm{P}$ and $\mathrm{Zr}$ are depleted in all samples. Similar enrichments of elements mobile in hydrous fluids (LILE), over those, which are considered immobile (HFSE), are typical of K-rich basalts derived from mantle domains influenced by subduction (Tatsumi - Eggins 1995 and references therein).

\section{$\mathrm{Sr}-\mathrm{Nd}$ isotopes}

Two newly obtained whole-rock $\mathrm{Sr}-\mathrm{Nd}$ isotopic ratios for the quartz monzodiorites, age-corrected to $290 \mathrm{Ma}$, are presented in Table 5 and plotted in Fig. 9. Even though the precise intrusion age for these rocks is unknown, the rather low $\mathrm{Rb} / \mathrm{Sr}$ and $\mathrm{Sm} / \mathrm{Nd}$ ratios render the calculated initial ratios rather insensitive to errors up to $\pm 20 \mathrm{Ma}$ ( \pm 0.00005 on ${ }^{87} \mathrm{Sr} /{ }^{86} \mathrm{Sr}_{\mathrm{i}}$ or $\pm 0.3 \varepsilon_{\mathrm{Nd}}$ units).

The $\mathrm{Nd}$ isotopic compositions of both samples are mutually comparable $\left(\varepsilon_{\mathrm{Nd}}^{290} \sim-2.6\right.$ and -3.1$)$, yielding correspondingly uniform single-stage $\mathrm{Nd}$ depleted-mantle model ages $\left(\mathrm{T}_{\mathrm{DM}}^{\mathrm{Nd}}=0.97-1.02 \mathrm{Ga}\right)$. Even closer to each other are the $\mathrm{Sr}$ isotopic signatures $\left({ }^{87} \mathrm{Sr} /{ }^{86} \mathrm{Sr}_{290}=0.7064\right.$ and 0.7066 ).

\section{Discussion}

Mode of emplacement

Owing to extensive cover of Upper Cretaceous, Neogene and Quaternary sediments and limited basement exposure it was not possible to study and characterize the local fault structures that were exploited by the intruding quartz monzodiorite dykes. Nevertheless, the notably finergrained texture of the quartz monzodiorites from Hamr and Č́tov, compared to the Štěpánovice dyke, may suggest that they form smaller and more rapidly cooled pulses. The rare occurrence of amygdaloidal variety of the pyroxene-biotite quartz monzodiorite documented from the borehole in the Skalice quarry indicates a rather shallow (subvolcanic) intrusion for the Štěpánovice dyke. The skeletal morphology of ilmenite and zircon lend further support to magma crystallization at high cooling rates, as does the presence of acicular apatite (Wyllie et al. 1962).

The field relations, overall geometry of the dyke and abundance of the white hydrothermal quartz xenoliths, in particular in the Skalice quarry, suggest emplacement of the dyke along a fault discontinuity partly filled by vein quartz.

Origin of the chlorite-actinolite spheroids

The chlorite-actinolite spheroids are rather difficult to interpret as to their primary composition. The most likely explanation is as pseudomorphs replacing former orthopyroxene or low-Ca augite. However, some interaction with the surrounding melt would be necessary to explain the aluminium enrichment (19.4 wt. \% $\mathrm{Al}_{2} \mathrm{O}_{3}$ ) characteristic of the chlorite.

\section{Significance of the Al-rich enclaves}

In the Al-rich enclaves, the corundum and spinel are thought to have formed mainly by consumption of sillimanite. The enclaves seem to represent refractory residua left after partial melting of small sillimanite-biotite 

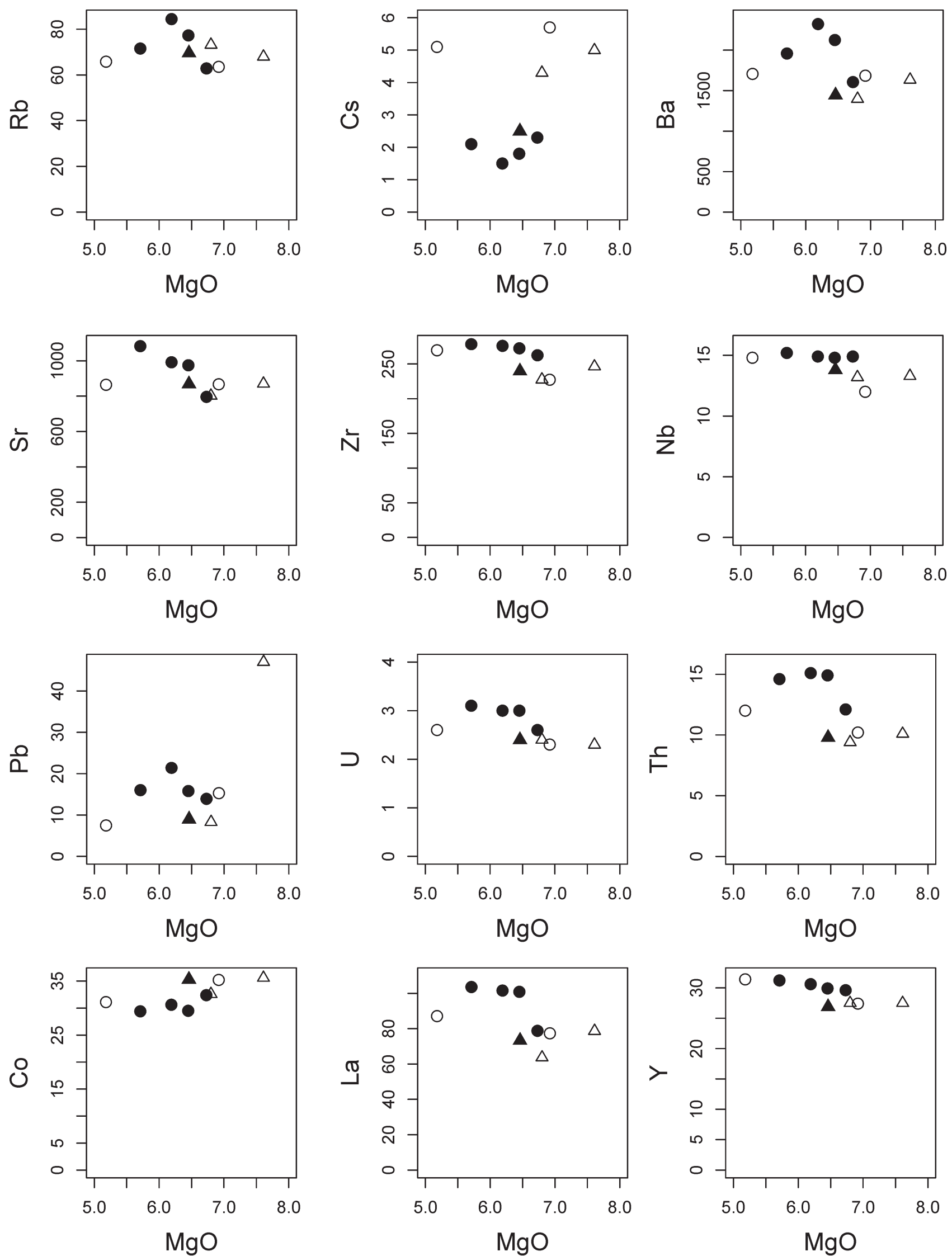

Skalice $\Delta$ Vlkovice OČítov $\triangle \mathrm{Hamr}$

Fig. 7 Binary diagrams of $\mathrm{MgO}$ versus selected trace elements (ppm). 
paragneiss xenoliths, corresponding therefore to surmicaceous enclaves sensu Montel et al. (1991). Although two-pyroxene mafic granulite dominates at the present surface around the Štěpánovice dyke, sillimanite-biotite paragneiss is the most abundant rock type on the regional scale. Thus it is likely that the metasediments could have been sampled by the monzodioritic magma at the depth. The amount of country rocks assimilated is uncertain, but probably low as indicated by limited variation of the whole-rock chemistry. Moreover the proportion of the Al-rich enclaves never exceeds 5 vol. \%, often much less, suggesting an insignificant role for contamination by metasedimentary material.

\section{Source of the parental magma}

For such basic, magnesian and isotopically relatively primitive magmas, a mantle source is clearly required at least when reasonable degrees of partial melting are to be assumed. Still, the exact nature of the primary magma parental to the quartz monzodiorite suite is poorly constrained. The dykes are fairly differentiated as shown by their rather high $\mathrm{SiO}_{2}$ and low mg\# (53-62), accompanied by elevated $\mathrm{FeOt} / \mathrm{MgO}$ ratios (1.10-1.57). For comparison, primary mantle-derived melts should have $\mathrm{mg \#} \sim 65-80$ and $\mathrm{FeOt} / \mathrm{MgO}$ ratios lower than unity (Rock 1991, Tatsumi - Eggins 1995).

The $\mathrm{Sr}-\mathrm{Nd}$ isotopic data, however limited they may be, seem to rule out a closed-system derivation from either Depleted Mantle or a mantle source isotopically similar to the Bulk Earth. Instead they require a slightly enriched mantle parentage and/or a significant contamination by crustal rocks. The similarity between the isotopic signatures of the samples Li-1 and Li-2 may argue for the first possibility, as does the limited variation in the whole-rock geochemical signature of the whole suite. The rather high $\mathrm{Pb}, \mathrm{U}$ and $\mathrm{Th}$ contents of the both samples are equivocal, as they can be equally well explained by a small-degree of enriched mantle melting, crustal contamination and/or passive enrichment due to fractional crystallization (strongly incompatible behaviour). Clearly much larger isotopic data set would be needed to constrain further the exact chemistry of the mantle domain involved.

\section{Geotectonic setting}

According to the current geotectonic models of the Variscan orogen in the Bohemian Massif, there has been an igneous arc active at the margin of the Moldanubian Zone in the relatively short period spanning the Late Devonian-Viséan times (c. 375-355 Ma: see Finger et al. 1997, Janoušek et al. 2006b for a review). Thus the likely Stephanian-Autunian age of the investigated quartz monzodiorite suite post-dated the subduction by as much as 50-75 Ma. The magmas were clearly emplaced already after the collision and subsequent orogenic collapse, in extensional regime.
However the dykes do not show a geochemical fingerprint of within-plate, post-orogenic magmas (alkaline chemistry, high contents of Fe and HFSE - e.g. Bonin et al. 1998). Instead, they give a (false) impression of an arc-related igneous suite: e.g., high-K calc-alkaline/shoshonitic chemistry, LREE/HREE enrichments and $\mathrm{Nb}-\mathrm{Ti}-$ $\mathrm{P}$ troughs in the primitive-mantle normalized spider plots, and the $\mathrm{Sr}-\mathrm{Nd}$ isotopic composition requiring a slightly enriched mantle source. Similar to the case of the volcanic Halle Complex, Germany (Romer et al. 2001), the geochemical signature of the dykes seems to be inherited from their source, rather than reflect the true geotectonic setting. The situation of the investigated quartz monzodioritic dykes located above the former orogenic root zone is thus in a sharp contrast with the basins closer to the outer bounds of the Variscan orogenic belt, where the subduction continued perhaps till the CarboniferousPermian boundary (e.g., Saar-Nahe Basin: Schmidberger - Hegner 1999).

In fact, hybrid high-K calc-alkaline rocks occur frequently in post-collisional uplift settings $\left(\mathrm{H}_{\mathrm{LO}}\right.$ group of Barbarin 1990). Such a magmatism is thought to have resulted from re-melting of lithosphere modified by subduction, and may post-date its cessation by as much as $50 \mathrm{Ma}$ depending on the availability of water (Bonin 1990). The generation of these magmas requires favourable regimes both thermal (thinned lithosphere, heated by upwelling asthenosphere) and tectonic (brittle, deep-seated strike-slip faulting facilitating the magma ascent; Liégeois et al. 1998).

\section{Genesis of the Late Variscan mafic dykes in the} Moldanubian Zone

The quartz monzodiorites examined in the present paper show some similarities to the Late Variscan Ševětín (Vrána et al. 1993) and Sušice (Vrána 2004) dyke swarms. They include the geological setting (intruding the deepseated, late extensional fractures, occasional occurrence of amygdaloidal types indicating a relatively shallow level of intrusion), petrology (fine-grained aphyric rocks, subophitic textures, presence of augite and evidence for magma quenching), as well as geochemical character (rather basic, shoshonitic/K-rich calc-alkaline rocks: Fig. 5, with mutually comparable trace-element patterns: Fig. 10).

Worth noting are the fairly primitive $\mathrm{Sr}-\mathrm{Nd}$ isotopic compositions of the Štěpánovice, Ševětín, and Sušice (this study, Vrána et al. 1993, Janoušek et al. 2002, Vrána 2004) dykes, strikingly different from the bulk of the Variscan magmatic rocks in the area, represented by the earlier, syn-collisional plutonic rocks of the South Bohemian Plutonic Complex (Gerdes 1997, Matějka - Janoušek 1998). The $\mathrm{Sr}-\mathrm{Nd}$ isotopic plot (Fig. 9) suggests that the composition of the late Variscan dykes in Southern Bohemia and the Pfahl granites in Lower Austria may reflect a mixing between two components: (1) a relatively primitive, mantle-derived magmas, and (2) a material 


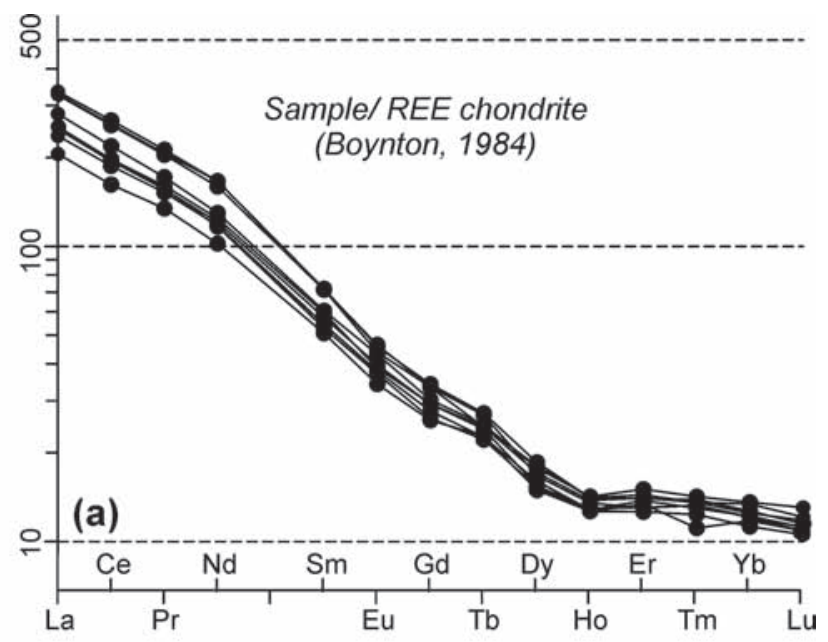

(b)
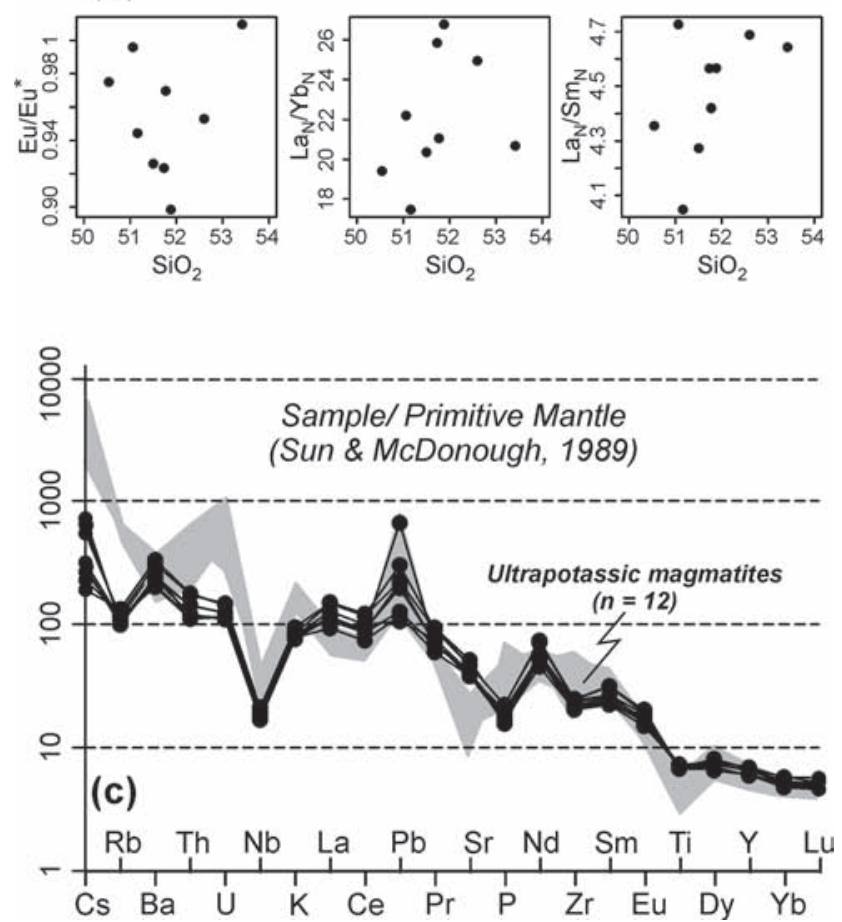

Fig. 8 a-Chondrite-normalized (Boynton 1984) REE patterns for the quartz monzodiorites; $\mathrm{b}$ - Binary plots of $\mathrm{SiO}_{2}$ versus parameters characterising the shape of the normalized REE patterns $\left(\mathrm{Eu} * / \mathrm{Eu}, \mathrm{La}_{\mathrm{N}} / \mathrm{Yb}_{\mathrm{N}}\right.$ and $\mathrm{La}_{\mathrm{N}} / \mathrm{Sm}_{\mathrm{N}}$ ). c - Primitive Mantle-normalized (Sun - McDonough 1989) spider plots for the same. The shaded field corresponds to 12 analyses of ultrapotassic rocks (durbachites and two-pyroxene melasyenites) from the Moldanubian Unit (Janoušek - Holub 2007).

corresponding to geochemically and isotopically significantly more evolved crustal rocks.

In order to place some constraints onto the nature of possible end-member compositions that could have been involved in such a mixing, we have undertaken a reverse modelling by the least-squares method (Albarède 1995; see Janoušek et al. 2002 for the R language code). The mixing hyperbola fits well most of the available data for the Štěpánovice dyke (this work), as well as the Ševětín (Vrána et al. 1993, Janoušek et al. 2002) and Sušice swarms (Vrána 2004). The only exception are two Sušice samples that seem to have been contaminated by a distinct material, having $\mathrm{Nd}$ isotopically similar to the Štěpánovice dykes but with $\mathrm{Sr}$ significantly more radiogenic $\left({ }^{87} \mathrm{Sr} /{ }^{86} \mathrm{Sr}_{290}>0.708\right)$, possibly reflecting hydrothermal alteration and, or interaction with the country-rock carbonates (the presence of calcite amygdales).

As the least-squares solution of the inverse mixing problem, we have obtained a hyperbola with asymptotes $\mathrm{x}=0.7057$ and $\mathrm{y}=-5.9$, and curvature factor $\mathrm{K}=0.0022$ (see Albarède 1995 for terminology and the underlying mathematic apparatus). The first of the asymptotes indicates that the basic end-member could not have been derived from Depleted Mantle. Instead, CHUR-like or even slightly enriched mantle source $\left({ }^{87} \mathrm{Sr} /{ }^{86} \mathrm{Sr}_{290}>0.7055\right.$, $\left.\varepsilon_{\mathrm{Nd}}^{290}>-2.5\right)$ is required. The crustal end-member had to have rather radiogenic $\mathrm{Sr}-\mathrm{Nd}$ isotopic signature $\left({ }^{87} \mathrm{Sr} /{ }^{86} \mathrm{Sr}_{290}>0.7095 ; \varepsilon_{\mathrm{Nd}}^{290}>-6\right)$. This means that the crustal end-member would need to be much more primitive than the typical Moldanubian paragneisses $\left(\varepsilon_{\mathrm{Nd}}^{290}\right.$ mostly lower than -8: Janoušek et al. 2002 and references therein.

Examining the Fig. 9, grossly similar scenario might be applicable also to explain the compositional spectrum observed in the late Variscan Pfahl and Mauthausen intrusions, Austria (Gerdes 1997). Indeed this author attributed an important role to enriched-mantle melts in the petrogenesis of the Mauthausen Group. Such a role could have been two-fold, as a source of the associated quartz

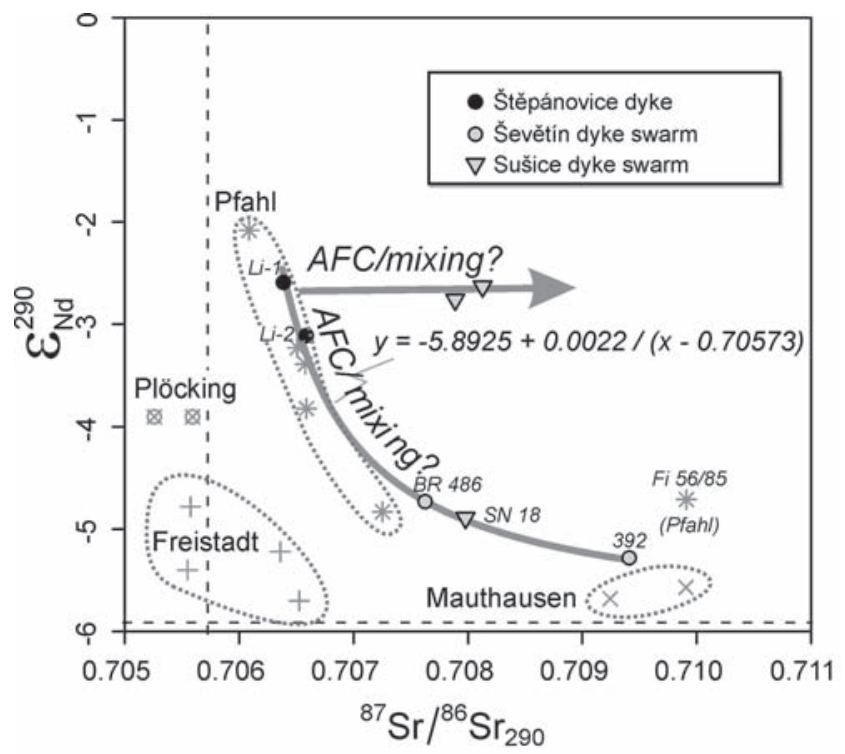

Fig. 9 A theoretical mixing hyperbola fitting data points for late Variscan Moldanubian basic-intermediate dyke swarms: Štěpánovice (this work), Ševětín (Vrána et al. 1993, Janoušek et al. 2002) and Sušice (Vrána 2004). Also plotted are analyses of the Mauthausen Group of the South Bohemian Plutonic Complex (Liew et al. 1989, Vellmer - Wedepohl 1994, Gerdes 1997). The asymptotes for the model hyperbola are shown by dashed lines. 


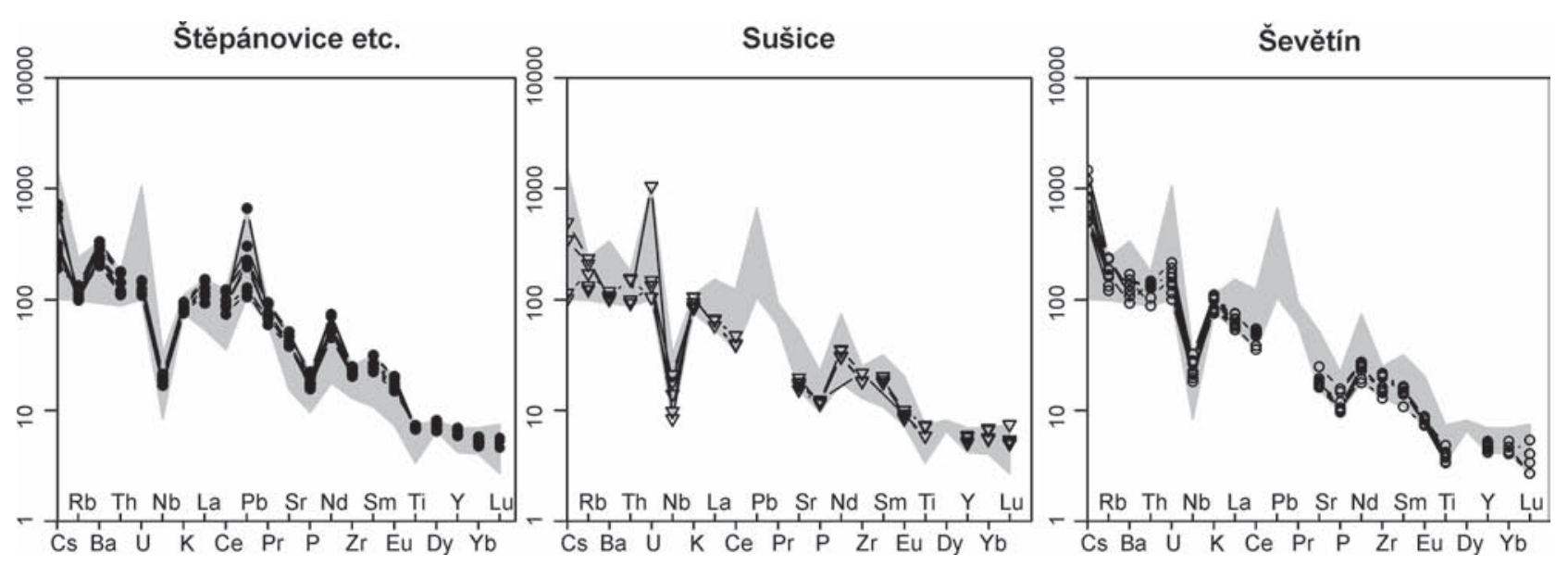

Fig. 10 Comparison of Primitive Mantle-normalized (Sun - McDonough 1989) spiderplots for the quartz monzodiorite dykes (this work), Ševětín (Vrána et al. 1993) and Sušice (Vrána 2004) swarms. The shaded field portrays the overall variability observed in the dataset.

dioritic rocks, and as a heat supply causing a widespread anatexis of a heterogeneous but at the same time quite primitive (tonalitic? - Gerdes 1997) crust.

In summary, the late (Stephanian-Autunian) basic-intermediate magmas parental to several dyke swarms in southern Bohemia, seem to have intruded in extensional regime, following the orogenic collapse. The crustal thinning at the waning stages of the orogeny could have led to upwelling and adiabatic decompression melting of the local, by the Late Devonian-Early Carboniferous subduction modified, lithospheric mantle. The dyke rocks that have penetrated into shallow levels of the brittle, freshly formed Variscan crust show an evidence for involvement of open-system processes. A major role in petrogenesis some of the swarms is ascribed to assimilation of crustal rocks/their melts by rather primitive, slightly enriched mantle-derived magmas similar to the quartz monzodiorites studied in the present contribution. The contaminant seems to have corresponded to crustal lithologies, geochemically and isotopically much less evolved than the paragneisses widespread at the current exposure level. The contamination is the most apparent in more fractionated, i.e. relatively $\mathrm{Sr}$-poor magmas.

\section{Conclusions}

1. Pyroxene-biotite quartz monzodiorite of the Štěpánovice dyke ( $3 \mathrm{~km}$ long and up to $50 \mathrm{~m}$ wide), together with additional small occurrences further north, are associated with the Late Variscan Blanice Graben.

2. The texture is subophitic, fine- to medium-grained, with skeletal crystals of ilmenite and zircon. This, together with rare amygdaloidal texture, indicates a rapid cooling of the melt emplaced at shallow (subvolcanic) crustal levels.

3. Quartz monzodiorites are metaluminous $(\mathrm{A} / \mathrm{CNK}=$ 0.79-0.86), high-K calc-alkaline rocks straddling the boundary to the shoshonitic domain. A characteristic feature in the Primitive-Mantle normalized spider plots are LREE/HREE enrichments as well as $\mathrm{Nb}-$
Ti-P troughs, resembling magmas derived in continental arc settings.

4. The $\mathrm{Nd}$ isotopic compositions of two samples $\left(\varepsilon_{\mathrm{Nd}}^{290}\right.$ $\sim-2.6$ and -3.1 ) correspond to single-stage Nd depleted-mantle model ages of 0.97 and $1.02 \mathrm{Ga}$; the strontium is unradiogenic $\left({ }^{87} \mathrm{Sr}^{86} \mathrm{Sr}_{290}=0.7064\right.$ and 0.7066$)$. As such they document the derivation of the parental magma from a slightly enriched mantle source.

5. The geological position, petrologic and whole-rock geochemical compositions of the studied quartz monzonite dykes resemble pyroxene microgranodiorite-quartz monzodiorite of the adjacent, $\sim 270 \mathrm{Ma}$ old Ševětín dyke swarm and the quartz micromonzodioritic Sušice dyke swarm further to the WNW.

6. The igneous arc-like geochemical signature of the dykes seems to be inherited from the source, rather than to reflect the true geotectonic setting. All these late Variscan (?Permian) basic-intermediate dykes seem to have intruded in extensional regime, following the orogenic collapse.

7. The primary magmas parental to the quartz monzodiorites originated most likely by decompression melting of the local, by the earlier subduction modified, lithospheric mantle. The dykes penetrated into shallow levels of the brittle Variscan crust. En route to the surface, they have suffered various degrees of assimilation by crustal material.

Acknowledgements. The authors thank D. Dolejs (Bayreuth) for his thorough review and helpful comments that improved the manuscript significantly. We are indebted to J. Trnková, V. Kopecký and J. Zeman for technical assistance in the isotope laboratory in Prague, V. Sixta (Czech Geological Survey Prague-Barrandov) for majorelement analyses. J. Malec operated the microprobe during a joint analytical work. This work has been supported by Czech Grant Agency. Further support from the Czech Geological Survey Internal Project 3248 is gratefully acknowledged.

Submitted November 28, 2006 


\section{References}

Albarède, F. (1995): Introduction to Geochemical Modeling. - Cambridge University Press, Cambridge, 543 pp.

Bacon, C. R. (1989): Crystallization of accessory phases in magmas by local saturation adjacent to phenocrysts. - Geochim. Cosmochim. Acta, 53: 1055-1066.

Barbarin, B. (1990): Granitoids: main petrogenetic classifications in relation to origin and tectonic setting. - Geol. J., 25: 227-238.

Benek, R. - Kramer, W. - McCann, T. - Scheck, M. - Negendank, J. F. W. - Korich, D. - Huebscher, H. D. - Bayer, U. (1996): Permo-Carboniferous magmatism of the Northeast German Basin. - Tectonophysics, 266: 379-404.

Bonin, B. (1990): From orogenic to anorogenic settings: evolution of granitoid suites after a major orogenesis. - Geol. J., 25: 261-270.

Bonin, B. - Azzouni-Sekkal, A. - Bussy, F. - Ferrag, S. (1998): Alkalicalcic and alkaline post-orogenic (PO) granite magmatism: petrologic constraints and geodynamic settings. - Lithos, 45: 45-70.

Boynton, W. V. (1984): Cosmochemistry of the rare earth elements: meteorite studies. - In: Henderson, P. (ed.) : Rare Earth Element Geochemistry. Elsevier, Amsterdam, 63-114.

Brandmayr, M. - Dallmeyer, R. D. - Handler, R. - Wallbrecher, E. (1995): Conjugate shear zones in the Southern Bohemian Massif (Austria): implications for Variscan and Alpine tectonothermal activity. Tectonophysics, 248: 97-116.

Cox, K. G. - Bell, J. D. - Pankhurst, R. J. (1979): The Interpretation of Igneous Rocks. - George Allen \& Unwin, $450 \mathrm{pp}$.

Debon, F - Le Fort, P. (1983): A chemical-mineralogical classification of common plutonic rocks and associations. - Trans. Roy. Soc. Edinb, Earth Sci., 73: 135-149.

- (1988): A cationic classification of common plutonic rocks and their magmatic associations: principles, method, applications. - Bull. Minéral., 111: 493-510.

Finger, F. - Roberts, M. P. - Haunschmid, B. - Schermaier, A. - Steyrer, H. P. (1997): Variscan granitoids of central Europe: their typology, potential sources and tectonothermal relations. - Min. Petrol., 61: 67-96.

Foley, S. F. - Venturelli, G. - Green, D. H. - Toscani, L. (1987): Ultrapotassic rocks: characteristics, classification and constraints for petrogenetic models. - Earth Sci. Rev., 24: 81-134.

Friedl, G. - von Quadt, A. - Frasl, G. - Finger, F. (1992): Neue U/Pb Altersdaten aus der südlichen Böhmischen Masse. - Frankfurter Geowiss. Arb., A11: 217-218.

Gerdes, A. (1997): Geochemische und thermische Modelle zur Frage der spätorogenen Granitgenese am Beispiel des Südböhmischen Batholiths: Basaltisches Underplating oder Krustenstapelung?- Unpublished $\mathrm{PhD}$. thesis, Georg-August-Universität, Göttingen, $113 \mathrm{pp}$.

Gerdes, A. - Friedl, G. - Parrish, R. R. - Finger, F. (2003): High-resolution geochronology of Variscan granite emplacement - the South Bohemian Batholith. - J. Czech Geol. Soc., 48: 53-54.

Hutchison, C. S. (1975): The norm, its variations, their calculation and relationships. - Schweiz. mineral. petrogr. Mitt., 55: 243-256.

Irvine, T. N. - Baragar, W. R. A. (1971): A guide to the chemical classification of the common volcanic rocks. - Can. J. Earth. Sci., 8: 523548.

Jacobsen, S. B. - Wasserburg, G. J. (1980): Sm-Nd evolution of chondrites. - Earth Planet. Sci. Lett., 50: 139-155.

Janoušek, V. - Holub, F. V. (2007): The causal link between HP-HT metamorphism and ultrapotassic magmatism in collisional orogens: case study from the Moldanubian Zone of the Bohemian Massif. - Proceedings of the Geologists' Association, 118: in press.

Janoušek, V. - Vrána, S. - Erban, V. (2002): Petrology, geochemical character and petrogenesis of a Variscan post-orogenic granite: case study from the Ševětín Massif, Moldanubian Batholith, Southern Bohemia. - J. Czech Geol. Soc., 47: 1-22.

Janoušek, V. - Farrow, C. M. - Erban, V. (2006a): Interpretation of wholerock geochemical data in igneous geochemistry: introducing Geochemical Data Toolkit (GCDkit). - Journal of Petrology, 47: 1255-1259.
Janoušek, V.-Gerdes, A. - Vrána, S. -Finger, F. Erban, V.-Friedl, G. - Braithwaite, C. J. R. (2006b): Low-pressure granulites of the Lišov Massif, Southern Bohemia: Viséan metamorphism of Late Devonian plutonic arc rocks. - Journal of Petrology, 47: 705-744.

Košler, J. - Kelley, S. P. - Vrána, S. (2001): ${ }^{40} \mathrm{Ar} /{ }^{39} \mathrm{Ar}$ hornblende dating of a microgranodiorite dyke: implications for early Permian extension in the Moldanubian Zone of the Bohemian Massif. - Int. J. Earth Sci. (Geol. Rundsch.), 90: 379-385.

Liégeois, J.-P. - Navez, J. - Hertogen, J. - Black, R. (1998): Contrasting origin of post-collisional high-K calc-alkaline and shoshonitic versus alkaline and peralkaline granitoids. - Lithos, 45: 1-28.

Liew, T. C. - Hofmann, A. W. (1988): Precambrian crustal components, plutonic associations, plate environment of the Hercynian Fold Belt of Central Europe: indications from a $\mathrm{Nd}$ and $\mathrm{Sr}$ isotopic study. Contrib. Mineral. Petrol., 98: 129-138.

Liew, T. C. - Finger, F. - Höck, V. (1989): The Moldanubian granitoid plutons in Austria: chemical and isotopic studies bearing on their environmental setting. - Chem. Geol., 76: 41-55.

Lugmair, G. W. - Marti, K. (1978): Lunar initial ${ }^{143} \mathrm{Nd} /{ }^{144} \mathrm{Nd}$ : differential evolution line of the lunar crust and mantle. - Earth Planet. Sci. Lett., 39: 349-357.

Matějka, D. - Janoušek, V. (1998): Whole-rock geochemistry and petrogenesis of granites from the northern part of the Moldanubian Batholith (Czech Republic). - Acta Univ. Carol., Geol., 42: 73-79.

Mattern, F. (2001): Permo-Silesian movements between Baltica and Western Europe: tectonics and ,basin families'. - Terra Nova, 13: 368375.

Montel, J. M. - Didier, J. - Pichavant, M. (1991): Origin of surmicaceous enclaves in intrusive granites. - In: Didier, J. - Barbarin, B. (eds) : Enclaves and Granite Petrology. Elsevier, Amsterdam, 509-528.

Peccerillo, A. - Taylor, S. R. (1976): Geochemistry of Eocene calc-alkaline volcanic rocks from the Kastamonu area, Northern Turkey. Contrib. Mineral. Petrol., 58: 63-81.

Richard, P. - Shimizu, N. - Allégre, C. J. (1976): ${ }^{143} \mathrm{Nd} /{ }^{146} \mathrm{Nd}$, a natural tracer: an application to oceanic basalts. - Earth Planet. Sci. Lett., 31: 269-278.

Rock, N. M. S. (1991): Lamprophyres. - Blackie, Glasgow and London, $285 \mathrm{pp}$.

Romer, R. L. - Förster, H. J. - Breitkreuz, C. (2001): Intracontinental extensional magmatism with a subduction fingerprint - the Late Carboniferous Halle Volcanic Complex (Germany). - Contrib. Mineral. Petrol., 141: 201-221.

Schmidberger, S. S. - Hegner, E. (1999): Geochemistry and isotope systematics of calc-alkaline volcanic rocks from the Saar-Nahe basin (SW Germany) - implications for Late-Variscan orogenic development. - Contrib. Mineral. Petrol., 135: 373-385.

Steiger, R. H. - Jäger, E. (1977): Subcommission on Geochronology; convention on the use of decay constants in geo- and cosmochronology. - Earth Planet. Sci. Lett., 36: 359-362.

Sun, S. S. - McDonough, W. F. (1989): Chemical and isotopic systematics of oceanic basalts: implications for mantle composition and processes. - In: Saunders, A. D. - Norry, M. (eds): Magmatism in Ocean Basins. Geological Society of London Special Publication 42, 313-345.

Tatsumi, Y. - Eggins, S. (1995): Subduction Zone Magmatism. - Frontiers in Earth Sciences, Blackwell, Cambridge, Mass., 211 pp.

Ulrych, J. - Pešek, J. - Štepánková-Svobodová, J. - Bosák, P. - Lloyd, F. E. - von Seckendorff, V. - Lang, M. - Novák, J. K. (2006): PermoCarboniferous volcanism in late Variscan continental basins of the Bohemian Massif (Czech Republic): geochemical characteristic. Chemie der Erde, 66: 37-56.

Vellmer, C. - Wedepohl, K. H. (1994): Geochemical characterization and origin of granitoids from the South Bohemian Batholith in Lower Austria. - Contrib. Mineral. Petrol., 118: 13-32.

Vrána, S. (2004): Late Variscan Sušice dyke swarm (Moldanubian Zone): quartz micromonzodiorite dykes and their pyroxene gabbro xenoliths. - Bull. Czech Geol. Survey, 79: 221-229.

Vrána, S. - Bártek, J. (2005): Retrograde metamorphism in a regional shear zone and related chemical changes: The Kaplice Unit of mus- 
covite-biotite gneisses in the Moldanubian Zone of southern Bohemia, Czech Republic. - J. Czech Geol. Soc., 50: 43-57.

Vrána, S. - Bendl, J. - Buzek, F. (1993): Pyroxene microgranodiorite dykes from the Ševětín structure, Czech Republic: mineralogical, chemical, and isotopic indication of a possible impact melt origin. - J. Czech Geol. Soc., 38: 129-148.
Vrána, S. - Slabý, J. - Bendl, J. (2005): The Kaplice dyke swarm of biotite granodiorite porphyry and its relationship to the Freistadt granodiorite, Moldanubian Batholith. - J. Czech Geol. Soc., 50: 9-17.

Wyllie, P. J. - Cox, K. G. - Biggar, G. M. (1962): The habit of apatite in synthetic systems and igneous rocks. - Journal of Petrology, 3: 238243.

\section{Pozdně orogenní variský magmatismus: žíly křemenného monzodioritu v blanické brázdě jižních Čech}

Pyroxen-biotitický křemenný monzodiorit štěpánovické žíly, 3 km dlouhé a až 50 m široké, je spolu s dalšími drobnými výskyty u Veselí nad Lužnicí vázán na zlomové pásmo blanické brázdy v moldanubiku jižních Čech. Jemnozrnná až středně zrnitá hornina má subofitickou strukturu a obsahuje kostrovité krystaly ilmenitu a zirkonu. Tyto vlastnosti a vzácná mandlovcovitá textura indikují rychlé zchlazení magmatu intrudovaného do mělké úrovně kůry. Křemenné monzodiority jsou subaluminické $(\mathrm{A} / \mathrm{CNK}=0.79-0.86)$, draslíkem bohaté alkalicko-vápenaté horniny s chemismem při hranici šošonitové domény. V diagramech s normalizací prvků hodnotami primitivního pláště jsou charakteristická navýšení poměru LREE/HREE a snížené hodnoty $\mathrm{Nb}-\mathrm{Ti}-\mathrm{P}$, podobně jako je tomu u hornin kontinentálních magmatických oblouků. Izotopické složení Nd ve dvou vzorcích (e $\sim-2.6$ a -3.1 ) odpovídá jednostupňovým modelovým stáŕím (počítaným vzhledem k ochuzenému plášti) 0.97 a 1.02 miliard let. Stroncium má neradiogenní izotopické složení $\left({ }^{87} \mathrm{Sr} /{ }^{86} \mathrm{Sr}=0.7064\right.$ a 0.7066$)$.

Studované žíly křemenného monzodioritu mají svou geologickou pozicí, petrologií a geochemickým složením blízký vztah k žílám pyroxenických mikrogranodioritů a křemenných monzodioritů ševětínského žilného roje (stáří 270 mil. let) a k pyroxenickým křemenným monzodioritům sušického žilného roje ca. 75 km dále k ZSZ. Je pravděpodobné, že všechny tyto porce bazických-intermediárních magmat pozdně variského (?permského) stáří intrudovaly v extenzním režimu, následujícím kolaps orogenní struktury. Doprovodné ztenčení kůry mělo za následek výzdvih a adiabatické dekompresní tavení lokálního litosférického pláště modifikovaného pozdně paleozoickou subdukcí. Izotopické složení Sr a Nd indikují různý stupeň asimilace krustálního materiálu relativně primitivním magmatem odvozeným ze slabě obohaceného pláštového zdroje. 